\title{
THE MAXIMAL SUBGROUPS AND THE COMPLEXITY OF THE FLOW SEMIGROUP OF FINITE (DI)GRAPHS
}

\author{
GÁBOR HORVÁTH, CHRYSTOPHER L. NEHANIV, \\ AND KÁROLY PODOSKI
}

Dedicated to John Rhodes on the occasion of his 80th birthday.

\begin{abstract}
The flow semigroup, introduced by John Rhodes, is an invariant for digraphs and a complete invariant for graphs. After collecting together previous partial results, we refine and prove Rhodes's conjecture on the structure of the maximal groups in the flow semigroup for finite, antisymmetric, strongly connected digraphs.

Building on this result, we investigate and fully describe the structure and actions of the maximal subgroups of the flow semigroup acting on all but $k$ points for all finite digraphs and graphs for all $k \geq 1$. A linear algorithm (in the number of edges) is presented to determine these so-called 'defect $k$ groups' for any finite (di)graph.

Finally, we prove that the complexity of the flow semigroup of a 2-vertex connected (and strongly connected di)graph with $n$ vertices is $n-2$, completely confirming Rhodes's conjecture for such (di)graphs.
\end{abstract}

\section{INTRODUCTION}

John Rhodes in [9] introduced the flow semigroup, an invariant for graphs and digraphs (that is, isomorphic flow semigroups correspond to isomorphic digraphs). In the case of graphs, this is a complete invariant determining the graph up to isomorphism. The flow semigroup is the semigroup of transformations of the vertices generated by elementary

Date: 30 July 2017.

2010 Mathematics Subject Classification. 20M20, 05C20, 05C25, $20 \mathrm{~B} 30$.

Key words and phrases. Rhodes's conjecture, flow semigroup of digraphs, Krohn-Rhodes complexity, complete invariants for graphs, invariants for digraphs, permutation groups.

The research was partially supported by the European Council under the European Union's Seventh Framework Programme (FP7/2007-2013)/ERC under grant agreements no. 318202 and no. 617747, by the MTA Rényi Institute Lendület Limits of Structures Research Group, the first author was partially supported by the Hungarian National Research, Development and Innovation Office (NKFIH) grant no. K109185 and grant no. FK124814, and the third author was funded by the National Research, Development and Innovation Office (NKFIH) Grant No. ERC_HU_15 118286. 
collapsings corresponding to the edges of the (di)graph. An elementary collapsing corresponding to the directed edge $u v$ is a map on the vertices moving $u$ to $v$ and acting as the identity on all other vertices. (See Section 2 for all the precise definitions.)

A maximal subgroup of this semigroup for a finite (di)graph $D=$ $\left(V_{D}, E_{D}\right)$ acts by permutations on all but $k$ of its vertices $(1 \leq k \leq$ $\left.\left|V_{D}\right|-1\right)$ and is called a "defect $k$ group". The set of defect $k$ groups of a (di)graph is also an invariant. For each fixed $k$, they are all isomorphic to each other in the case of (strongly) connected (di)graphs. Rhodes formulated a conjecture on the structure of these groups for strongly connected digraphs whose edge relation is anti-symmetric in [9, Conjecture 6.51i (2)-(4)]. We show that his conjecture was correct, and we prove it here in sharper form. Moreover, extending this result, we fully determine the defect $k$ groups for all finite graphs and digraphs.

Rhodes further conjectured [9, Conjecture 6.51i (1)] that the KrohnRhodes complexity of the flow semigroup of a strongly connected, antisymmetric digraph $D$ on $n$ vertices is $n-2$. We confirm this conjecture when the digraph is 2-vertex connected, and bound the complexity in the remaining cases.

The structure of the argument is as follows. First, a maximal group in the flow semigroup of a digraph $D$ is the direct product of maximal groups of the flow semigroups of its strongly connected components. Thus one needs only to consider strongly connected digraphs. It turns out, that if $D$ is a strongly connected digraph, then the defect $k$ group (up to isomorphism) does not depend on the choice of the vertices it acts on. Furthermore, for a strongly connected digraph, its flow semigroup is the same as the flow semigroup of the simple graph obtained by "forgetting" the direction of the edges. This is detailed in Section 2 and is based on [9, p. 159-169]. Thus, one only needs to consider the defect $k$ groups of the flow semigroup for simple connected graphs.

In Section 3 we list some useful lemmas and determine the defect $k$ group of a cycle. In Section 4 we prove that the defect 1 group of arbitrary simple connected graph is the direct product of the defect 1 groups of its 2-vertex connected components. The defect 1 group of an arbitrary 2-vertex connected graph $\Gamma$ has been determined by Wilson [15]. He proved that the defect 1 group is either $A_{n-1}$ or $S_{n-1}$, unless $\Gamma$ is a cycle or the exceptional graph displayed in Figure 1.

In particular, Rhodes's conjecture (as phrased for strongly connected, antisymmetric digraphs in [9, Conjecture 6.51i (2)]) about the defect 1 group holds, and more generally: the defect 1 group of the flow semigroup of a simple connected graph is indeed the product of cyclic, alternating and symmetric groups of various orders. A straightforward linear algorithm is given to determine the direct components of the defect 1 group of an arbitrary connected graph (see Section 6). 


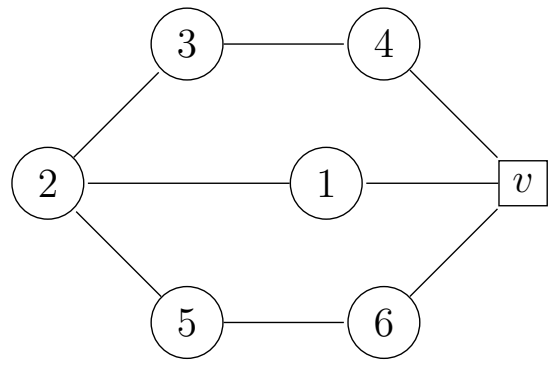

FiguRE 1. Exceptional graph

In Section 5 we determine the defect $k$ groups $(k \geq 2)$ of arbitrary graphs by considering the so-called maximal $k$-subgraphs (maximal subgraphs for which the defect $k$ group is the full symmetric group) and prove that the defect $k$ group of a graph is the direct product of the defect $k$ groups of the maximal $k$-subgraphs (i.e. of full symmetric groups). In Section 6 we provide a linear algorithm (in the number of edges of $\Gamma$ ) to determine the maximal $k$-subgraphs of an arbitrary connected graph. Finally, in Section 7 we confirm [9, Conjecture 6.51i (1)] about the Krohn-Rhodes complexity of digraphs when the digraph is 2 -vertex connected, and we prove some bounds on the complexity of the flow semigroup in the remaining cases. (See Section 7 for the definition of Krohn-Rhodes complexity.)

We have collected all these results into the following main theorem.

\section{Theorem 1.}

(1) Let $D$ be a digraph, then every maximal subgroup of $S_{D}$ is (isomorphic to) the direct product of maximal subgroups of $S_{D_{i}}$, where the $D_{i}$ are the strongly connected components of $D$.

(2) Let $D$ be a strongly connected digraph. Let $V_{k}, V_{k}^{\prime} \subseteq D$ be subsets of nodes such that $\left|V_{k}\right|=\left|V_{k}^{\prime}\right|=k$. Let $G_{k, V_{k}}, G_{k, V_{k}^{\prime}}$ be the defect $k$ groups acting on $V \backslash V_{k}$ and $V \backslash V_{k}^{\prime}$, respectively. Then $G_{k, V_{k}} \simeq G_{k, V_{k}^{\prime}}$ as permutation groups.

$\left(2^{\mathrm{r}}\right)$ Let $D$ be a strongly connected digraph, and $\Gamma_{D}$ be the graph obtained from $D$ by forgetting the direction of the edges in $D$. Then $S_{D}=S_{\Gamma_{D}}$.

(3) Let $\Gamma$ be a simple connected graph of $n$ vertices, and let $\Gamma_{1}, \ldots, \Gamma_{m}$ be its 2-vertex connected components. Then the defect 1 group of $\Gamma$ is the direct product of the defect 1 groups of $\Gamma_{i}(1 \leq i \leq m)$.

(4) Let $\Gamma$ be a 2-vertex connected simple graph with $n \geq 2$ vertices. Then the defect 1 group of $\Gamma$ is isomorphic (as a permutation group) to

(a) the cyclic group $Z_{n-1}$ if $\Gamma$ is a cycle;

(b) $S_{5} \simeq P G L_{2}(5)$ acting sharply 3-transitively on 6 points, if $\Gamma$ is the exceptional graph (see Figure 1); 
(c) $S_{n-1}$ or $A_{n-1}$, otherwise, where the defect 1 group is $A_{n-1}$ if and only if $\Gamma$ is bipartite.

$\left(4^{\mathrm{c}}\right)$ Let $\Gamma$ be a 2-vertex connected simple graph with $n \geq 2$ vertices. Then the complexity of $S_{\Gamma}$ is $\#_{G}\left(S_{\Gamma}\right)=n-2$.

$\left(4^{\mathrm{cc}}\right)$ Let $\Gamma$ be a 2-edge connected simple graph with $n \geq 2$ vertices. Then for the complexity of $S_{\Gamma}$ we have $n-3 \leq \#_{G}\left(S_{\Gamma}\right) \leq n-2$.

(5) Let $k \geq 2, \Gamma$ be a simple connected graph of $n$ vertices, $n>k$.

(a) If $\Gamma$ is a cycle, then its defect $k$ group is the cyclic group $Z_{n-k}$.

(b) Otherwise, let $\Gamma_{1}, \ldots, \Gamma_{m}$ be the maximal $k$-subgraphs of $\Gamma$, and let $\Gamma_{i}$ have $n_{i}$ vertices. Then the defect $k$ group of $\Gamma$ is the direct product of the defect $k$ groups of $\Gamma_{i}(1 \leq i \leq m)$, thus it is isomorphic (as a permutation group) to

$$
S_{n_{1}-k} \times \cdots \times S_{n_{m}-k} .
$$

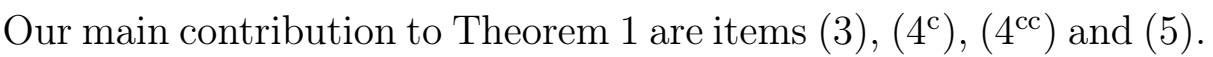
Items (11), (21) and (2 $\left.\mathrm{2}^{\mathrm{r}}\right)$ (among some basic definitions and notations) are detailed in Section 2 and are based on [9, p. 159-169]. In Section 3 we list some useful lemmas and determine the defect $k$ group of a cycle. Item (3) is proved in Section 4, while item (4) has already been proved by Wilson [15]. Then in Section 5 we prove item (5). In Section 6 we provide a linear algorithm (in the number of edges of $\Gamma$ ) to determine the maximal $k$-subgraphs of an arbitrary connected graph to help putting item (5) more into context. Finally, items $\left(4^{\mathrm{c}}\right)$ and $\left(4^{\mathrm{cc}}\right)$ are proved in Section 7.

East, Gadouleau and Mitchell [6] are currently looking into other properties of flow semigroups. In particular, they provide a linear algorithm (in the number of vertices of a digraph) for whether or not the flow semigroup contains a cycle of length $m$ for a fixed positive integer $m$. Furthermore, they classify all those digraphs whose flow semigroups have any of the following properties: inverse, completely regular, commutative, simple, 0 -simple, a semilattice, a rectangular band, congruence-free, is $\mathcal{K}$-trivial or $\mathcal{K}$-universal, where $\mathcal{K}$ is any of Green's $\mathcal{H}-, \mathcal{L}_{-}, \mathcal{R}-$, or $\mathcal{J}$-relation, and when the flow-semigroup has a left, right, or two-sided zero.

Rhodes's original conjecture [9, Conjecture 6.51i] is about strongly connected, antisymmetric digraphs. By [11] a strongly connected antisymmetric digraph becomes a 2-edge connected graph after forgetting the directions. Therefore Theorem 10 almost completely settles Rhodes's conjecture [9, Conjecture 6.51i]. To completely settle the last remaining part of Rhodes's conjecture [9, Conjecture 6.51i (1)], one should find the complexity of the flow semigroups for the rest of the 2-edge connected graphs.

Problem 1. Determine the complexity of $S_{\Gamma}$ for a 2-edge connected graph $\Gamma$ which is not 2 -vertex connected. 
The smallest such graph is the "bowtie" graph:

Problem 2. Let $\Gamma$ be the graph with vertex set $\{u, v, w, x, y\}$ and edge set $\{u v, v w, w u, w x, x y, y w\}$. Determine the complexity of $S_{\Gamma}$.

Ultimately, the goal is the determine the complexity for all flow semigroups.

Problem 3. Determine the complexity of $S_{\Gamma}$ for an arbitrary finite graph (or digraph) $\Gamma$.

\section{FLOW SEMIGROUP OF DIGRAPHS}

For notions in graph theory we refer to [4, 7], in group theory to [12 in permutation groups to [1, 5], in semigroup theory to [2, 3].

A semigroup is a set with a binary associative multiplication. A transformation on a set $X$ is a function $s: X \rightarrow X$. It operates (or acts) on $X$ by mapping each $x \in X$ to some $x \cdot s \in X$. Here we write $x \cdot s$ or $x s$ for transformation $s$ applied to $x \in X$. A transformation semigroup $S$ is a set of transformations $s \in S$ on some set $X$ such that $S$ is closed under (associative) function composition. Also, $S$ itself is then said to operate or to act on the set $X$. Note that in this paper functions act on the right, therefore transformations are multiplied from left to right. Denoting by $s s^{\prime}$ the transformation of $X$ obtained by first applying $s$ and then $s^{\prime}$, we have $x \cdot s s^{\prime}=(x \cdot s) \cdot s^{\prime}$. If a semigroup element $s$ acts on a set $X$, and for some $Y \supseteq X$ the action of $s$ is not defined on $Y \backslash X$, then we may consider $s$ acting on $Y$, as well, with the identity action on $Y \backslash X$.

A permutation group is a nonempty transformation semigroup $G$ that contains only permutations and such that that if $g \in G$ then the inverse permutation $g^{-1}$ is also in $G$. Furthermore, for a set $Y \subseteq X$ and a transformation $s$ on $X$ define

$$
Y s=\{y s \mid y \in Y\} .
$$

A subgroup $G$ of a transformation semigroup $S$ is a subset of $S$ whose transformations satisfy the (abstract) group axioms. It is not hard to show that if $S$ is a transformation semigroup acting on $X$, then $G$ contains a (unique) idempotent $e^{2}=e$ (which does not generally act as the identity map on $X$ ), and furthermore distinct elements of $G$ when restricted to $X e$ are distinct, permute $X e$, and comprise a permutation group acting on $X e$ (see [9, p. 49]).

A digraph $(V, E)$ is a set of nodes (or vertices) $V$, and a binary relation $E \subseteq V \times V$. An element $e=(u, v) \in E$ is called a directed edge from node $u$ to node $v$, and also denoted $u v$. A loop-edge is an edge from a vertex to itself. A graph $(V, E)$ is a set of nodes $V$ and a symmetric binary relation $E \subseteq V \times V$. If $(u, v) \in E$, then $u v$ is called an (undirected) edge. Such a graph is called simple if it has no loop-edges. In this paper we consider only digraphs without loop-edges 
and simple graphs. A walk is a sequence of vertices $\left(v_{1}, \ldots, v_{n}\right)$ such that $v_{i} v_{i+1}$ is a (directed) edge for all $1 \leq i \leq n-1$. By cycle we will mean a simple cycle, that is a closed walk with no repetition of vertices except for the starting and ending vertex. A path is a walk with no repetition of vertices. A (di)graph $\Gamma=(V, E)$ is (strongly) connected if there is a path from $u$ to $v$ for all distinct $u, v \in V$. By subgraph $\Gamma^{\prime}=\left(V^{\prime}, E^{\prime}\right) \subseteq \Gamma$ we mean a graph for which $V^{\prime} \subseteq V, E^{\prime} \subseteq E$. If $\Gamma^{\prime}$ is an induced subgraph, that is $E^{\prime}$ consists of all edges from $E$ with both endpoints in $V^{\prime}$, then we explicitly indicate it. A strongly connected component of a digraph $\Gamma$ is a maximal strongly connected subgraph of $\Gamma$.

For a digraph $D=\left(V_{D}, E_{D}\right)$ without any loop-edges, the flow semigroup $S=S_{D}$ is the semigroup of transformations acting on $V_{D}$ defined by

$$
S=S_{D}=\left\langle e_{u v} \mid u v \in E_{D}\right\rangle
$$

where $e_{u v}$ is the elementary collapsing corresponding to the directed edge $u v \in E_{D}$, that is, for every $x \in V_{D}$ we have

$$
x \cdot e_{u v}=x e_{u v}= \begin{cases}v, & \text { if } x=u, \\ x, & \text { otherwise }\end{cases}
$$

Thus, the flow semigroup of a (di)graph $D$ is generated by idempotents (elementary collapsings) corresponding to the edges of $D$. The flow semigroup $S_{D}$ is also called the Rhodes semigroup of the (di)graph.

A maximal subgroup of $S_{D}$ is a subgroup that is not properly contained in any other subgroup of $S_{D}$. In order to determine the maximal subgroups of $S_{D}$, one can make several reductions by [9, Proposition 6.51f]. First, one only needs to consider the maximal subgroups of $S_{D_{i}}$ for the strongly connected components $D_{i}$ of $D$. Strongly connected components are maximal induced subgraphs such that any vertex can be reached from any other vertex by a directed path.

Lemma 2 ([9, Proposition 6.51f (1)]). Let $D$ be a digraph, then every maximal subgroup of $S_{D}$ is (isomorphic to) the direct product of maximal subgroups of $S_{D_{i}}$, where the $D_{i}$ are the strongly connected components of $D$.

This is (11) of Theorem 1, An element $s \in S$ is of defect $k$ if $\left|V_{D} s\right|=$ $\left|V_{D}\right|-k$. Let $V_{k}=\left\{v_{1}, v_{2}, \ldots, v_{k}\right\} \subseteq V_{D}$. The defect $k$ group $G_{k, V_{k}}$ associated to $V_{k}$ (called the defect set) is generated by all elements of $S$ restricted to $V_{D} \backslash V_{k}$ which permute the elements of $V_{D} \backslash V_{k}$ and move elements of $V_{k}$ to elements of $V_{D} \backslash V_{k}$ :

$$
G_{k, V_{k}}=\left\langle s\left\lceil_{V_{D} \backslash V_{k}}: s \in S,\left(V_{D} \backslash V_{k}\right) s=V_{D} \backslash V_{k}, V_{k} s \subseteq V_{D} \backslash V_{k}\right\rangle,\right.
$$

where $s \uparrow_{V_{D} \backslash V_{k}}$ denotes the restriction of the transformation $s$ onto the set $V_{D} \backslash V_{k}$. Now, $G_{k, V_{k}}$ is a permutation group acting on $V_{D} \backslash V_{k}$. 
For this reason $V_{D} \backslash V_{k}$ is called the permutation set of $G_{k, V_{k}}$, and the elements of $G_{k, V_{k}}$ are sometimes called defect $k$ permutations. Furthermore, if the defect set contains only one vertex $v$, then by abuse of notation we write defect $v$ or defect point $v$ instead of defect $\{v\}$. In general, the defect $k$ group $G_{k, V_{k}}$ can depend on the choice of $V_{k}$. However, by [9, Proposition 6.51f (2)] it turns out that if the graph is strongly connected then the defect $k$ group $G_{k}$ is unique up to isomorphism.

Lemma 3 ([9, Proposition 6.51f (2)]). Let D be a strongly connected digraph. Let $V_{k}, V_{k}^{\prime} \subseteq V_{D}$ be subsets of nodes such that $\left|V_{k}\right|=\left|V_{k}^{\prime}\right|=k$. Then the action of $G_{k, V_{k}}$ on $V_{D} \backslash V_{k}$ is equivalent to that of $G_{k, V_{k}^{\prime}}$ on $V_{D} \backslash V_{k}^{\prime}$. That is, $G_{k, V_{k}} \simeq G_{k, V_{k}^{\prime}}$ as permutation groups.

This is (2) of Theorem 1. By Lemma 3, we may write $G_{k}$ instead of $G_{k, V_{k}}$ without any loss of generality. Furthermore, the case of strongly connected graphs can be reduced to the case of simple graphs. Let $\Gamma=(V, E)$ be a simple (undirected) graph, we define $S_{\Gamma}$ by considering $\Gamma$ as a directed graph where every edge is directed both ways. Namely, let $D_{\Gamma}=\left(V, E_{D}\right)$ be the directed graph on vertices $V$ such that both $u v \in E_{D}$ and $v u \in E_{D}$ if and only if the undirected edge $u v \in E$. Then let $S_{\Gamma}=S_{D_{\Gamma}}$.

Furthermore, for every digraph $D=\left(V_{D}, E_{D}\right)$, one can associate an undirected graph $\Gamma$ by "forgetting" the direction of edges in $D$. Precisely, let $\Gamma_{D}=\left(V_{D}, E\right)$ be the undirected graph such that $u v \in E$ if and only if $u v \in E_{D}$ or $v u \in E_{D}$. The following lemma due to Nehaniv and Rhodes shows that if a digraph $D$ is strongly connected then the semigroup $S_{D}$ corresponding to $D$ and the semigroup $S_{\Gamma_{D}}$ corresponding to the simple graph $\Gamma_{D}$ are the same. Moreover, Lemma 4 immediately implies that the transformation semigroup $S_{D}$ is an invariant for digraphs and a complete invariant for (simple) graphs: That is, isomorphic digraphs have the isomorphic flow semigroups, and graphs are isomorphic if and only if their flow semigroups are isomorphic as transfromation semigroups.

Lemma 4 ([9, Lemma 6.51b]). Let $D$ be an arbitrary digraph. Then

$$
e_{a b} \in S_{D} \Longleftrightarrow\left\{\begin{array}{l}
a \rightarrow b \text { is an edge in } D \text {, or } \\
b \rightarrow a \text { is an edge in a directed cycle in } D .
\end{array}\right.
$$

In particular, if $D$ is strongly connected then $S_{D}=S_{\Gamma_{D}}$.

Proof. Let $b \rightarrow a \rightarrow u_{1} \rightarrow \cdots \rightarrow u_{n-1} \rightarrow b$ be a directed cycle in $D$. Then an easy calculation shows that

$$
e_{a b}=\left(e_{b a} e_{u_{n-1} b} e_{u_{n-2} u_{n-1}} \ldots e_{u_{1} u_{2}} e_{a u_{1}}\right)^{n} .
$$

For the other direction, assume $e_{a b}=e_{u v} s$ for some $s \in S_{D}$. Then $e_{u v} s$ moves $u$ and $v$ to the same vertex, while $e_{a b}$ moves only $a$ and $b$ to the same vertex. Thus $\{a, b\}=\{u, v\}$. 
This is $\left(2^{\mathrm{r}}\right)$ of Theorem 1. Therefore, in the following we only consider simple, connected, undirected graphs $\Gamma=(V, E)$, that is no selfloops or multiple edges are allowed. Furthermore, $\Gamma$ is 2-edge connected if removing any edge does not disconnect $\Gamma$. Rhodes's conjecture [9, Conjecture 6.51i (2)-(4)] is about strongly connected, antisymmetric digraphs. Note that by [11] a strongly connected antisymmetric digraph becomes a 2-edge connected graph after forgetting the directions.

Let us fix some notation. The letters $k, l, m$ and $n$ will denote nonnegative integers. The number of vertices of $\Gamma$ is usually denoted by $n$, while $k$ will denote the size of the defect set. Usually we denote the defect $k$ group of a graph $\Gamma$ by $G_{k}$ or $G_{\Gamma}$, depending on the context. We try to heed the convention of using $u, v, w, x, y$ as vertices of graphs, $V$ as the set of vertices, $E$ as the set of edges. Furthermore, the flow semigroup is mostly denoted by $S$, its elements are denoted by $s, t, g$, $h, p, q$. The cyclic group of $m$ elements is denoted by $Z_{m}$.

We will need the notion of an open ear, and open ear decomposition.

Definition 5. Let $\Gamma$ be an arbitrary graph, and let $\Gamma^{\prime}$ be a proper subgraph of $\Gamma$. A path $\left(u, c_{1}, \ldots, c_{m}, v\right)$ is called a $\Gamma^{\prime}$-ear (or open ear) with respect to $\Gamma$, if $u, v \in \Gamma^{\prime}, u \neq v$, and either $m=0$ and the edge $u v \notin \Gamma^{\prime}$, or $c_{1}, \ldots, c_{m} \in \Gamma \backslash \Gamma^{\prime}$. An open ear decomposition of a graph is a partition of its set of edges into a sequence of subsets, such that the first element of the sequence is a cycle, and all other elements of the sequence are open ears of the union of the previous subsets in the sequence.

A connected graph $\Gamma$ with at least $k$ vertices is $k$-vertex connected if removing any $k-1$ vertices does not disconnect $\Gamma$. By [14] a graph is 2 -vertex connected if and only if it is a single edge or it has an open ear decomposition.

\section{Preliminaries}

Let $\Gamma=(V, E)$ be a simple, connected (undirected) graph, and for every $1 \leq k \leq|V|-1$, let $G_{k}$ denote its defect $k$ group for some $V_{k} \subseteq V$, $\left|V_{k}\right|=k$. Let $S=S_{\Gamma}$ be the flow semigroup of $\Gamma$. The following is immediate.

Lemma 6 ([9, Fact 6.51c]). Let $s \in S$ be of defect $k$. If $s_{u v}$ is of defect $k$, as well, then $u \notin V s$ or $v \notin V s$.

Furthermore, it is not too hard to see that every defect 1 permutation arises from the permutations generated by cycles (in the graph) containing the defect point.

Lemma 7 ([9, Proposition 6.51e]). Let $\Gamma$ be a connected graph, and let $G_{1}$ denote its defect 1 group, such that the defect point is $v \in V$. Then

$$
\left.G_{1}=\left\langle\left(u_{1}, \ldots, u_{k}\right) \text { as permutation }\right|\left(u_{1}, \ldots, u_{k}, v\right) \text { is a cycle in } \Gamma\right\rangle \text {. }
$$


These yield that the defect $k$ group of the $n$-cycle graph is cyclic, proving items (4a) and (5a) of Theorem 1:

Lemma 8. The defect $k$ group of the $n$-cycle is isomorphic to $Z_{n-k}$.

Proof. Let $x_{1}, x_{2}, \ldots x_{n}$ be the consecutive elements of the cycle $\Gamma=$ $(V, E)$. If $s \in S$ is an element of defect $k$ then by Lemma 6 we have that $s e_{x_{i} x_{i+1}}$ is of defect $k$ if and only if $x_{i} \notin V s$ or $x_{i+1} \notin V s$. This means that if $u_{1}, u_{2}, \ldots u_{n-k}$ are the consecutive elements of $V s$ in the cycle and $s e_{x_{i} x_{i+1}}$ is of defect $k$, as well, then

$$
u_{1} e_{x_{i} x_{i+1}}, u_{2} e_{x_{i} x_{i+1}}, \ldots, u_{n-k} e_{x_{i} x_{i+1}}
$$

are the consecutive elements of $V s e_{x_{i} x_{i+1}}$. Thus the cyclic ordering of these elements cannot be changed. Hence $G_{k}$ is isomorphic to a subgroup of $Z_{n-k}$.

Now, assume that $v_{1}, v_{2}, \ldots v_{k}, u_{1}, u_{2}, \ldots u_{n-k}$ are the consecutive elements of $\Gamma$, and the defect set is $V_{k}=\left\{v_{1}, \ldots, v_{k}\right\}$. Let

$$
\begin{aligned}
s_{1} & =e_{v_{1} v_{2}} \ldots e_{v_{j} v_{j+1}} \ldots e_{v_{k-1} v_{k}}, \\
s_{2} & =e_{u_{n-k} v_{k}} e_{u_{n-k-1} u_{n-k}} \ldots e_{u_{j-1} u_{j}} \ldots e_{u_{1} u_{2}} e_{v_{k} u_{1}}, \\
s & =s_{1} s_{2} .
\end{aligned}
$$

It easy to check that

$$
v_{i} s=u_{1}, \quad u_{1} s=u_{2}, \ldots, u_{j} s=u_{j+1}, \ldots, u_{n-k} s=u_{1} .
$$

Therefore $s, s^{2}, \ldots, s^{n-k}$ are distinct elements of $G_{k}$, hence $G_{k} \simeq Z_{n-k}$.

\section{Defect 1 groups}

In this Section we prove item (3) of Theorem 1, which states that the defect 1 group of a simple connected graph is the direct product of the defect 1 groups of its 2 -vertex connected components. This follows by induction on the number of 2 -vertex connected components from Lemma 9. The case where $\Gamma$ is 2 -vertex connected (that is item (4) of Theorem 11) is covered by [15, Theorem 2].

Lemma 9. Let $\Gamma_{1}$ and $\Gamma_{2}$ be connected induced subgraphs of $\Gamma$ such that $\Gamma_{1} \cap \Gamma_{2}=\{v\}$, where there are no edges in $\Gamma$ between $\Gamma_{1} \backslash\{v\}$ and $\Gamma_{2} \backslash\{v\}$. Then the defect 1 group of $\Gamma_{1} \cup \Gamma_{2}$ is the direct product of the defect 1 groups of $\Gamma_{1}$ and $\Gamma_{2}$.

Proof. Let $G_{\Gamma_{i}}$ denote the defect 1 group of $\Gamma_{i}$, where the defect point is $v$. By Lemma 7, $G_{\Gamma}$ is generated by cyclic permutations corresponding to cycles through $v$ in $\Gamma$. Now, $\Gamma_{1} \cap \Gamma_{2}=\{v\}$, and every path between a node from $\Gamma_{1}$ and a node from $\Gamma_{2}$ must go through $v$, hence every cycle in $\Gamma$ is either in $\Gamma_{1}$ or in $\Gamma_{2}$. Let $c_{i}^{(1)}, \ldots, c_{i}^{\left(m_{i}\right)}$ be the permutations corresponding to the cycles in $\Gamma_{i}(i=1,2)$. Since these cycles do not 
involve $v$ by Lemma $\left[\right.$, we have $c_{1}^{\left(j_{1}\right)} c_{2}^{\left(j_{2}\right)}=c_{2}^{\left(j_{2}\right)} c_{1}^{\left(j_{1}\right)}$ for all $1 \leq j_{i} \leq m_{i}$, $i=1,2$, thus

$$
\begin{aligned}
G_{\Gamma}=\left\langle c_{1}^{(1)}, \ldots,\right. & \left.c_{1}^{\left(m_{1}\right)}, c_{2}^{(1)}, \ldots, c_{2}^{\left(m_{2}\right)}\right\rangle \\
& =\left\langle c_{1}^{(1)}, \ldots, c_{1}^{\left(m_{1}\right)}\right\rangle \times\left\langle c_{2}^{(1)}, \ldots, c_{2}^{\left(m_{2}\right)}\right\rangle=G_{\Gamma_{1}} \times G_{\Gamma_{2}} .
\end{aligned}
$$

\section{DefECT $k$ GROUPS}

We prove item (5b) of Theorem 1 in this Section. In the following we assume $k \geq 2$, and every graph $\Gamma$ is assumed to be simple connected. We start with some simple observations.

Lemma 10. Let $\Gamma$ be a connected graph, and let $\Gamma^{\prime}$ be a connected subgraph of $\Gamma$. If $\Gamma^{\prime}$ has at least $k+1$ vertices, then the defect $k$ group of $\Gamma$ contains a subgroup isomorphic (as a permutation group) to the defect $k$ group of $\Gamma^{\prime}$. Furthermore, if $\Gamma \backslash \Gamma^{\prime}$ contains at least one vertex, and $\Gamma^{\prime}$ has at least $k$ vertices, then the defect $k$ group of $\Gamma$ contains a subgroup isomorphic (as a permutation group) to the defect $k-1$ group of $\Gamma^{\prime}$.

Proof. Let $\Gamma=(V, E), \Gamma^{\prime}=\left(V^{\prime}, E^{\prime}\right)$. First, assume $\left|V^{\prime}\right| \geq k+1$, and let $V_{k}=\left\{v_{1}, \ldots, v_{k}\right\} \subseteq V^{\prime}$. Let $G_{k, V_{k}}$ and $G_{k, V_{k}}^{\prime}$ be the defect $k$-groups of $\Gamma$ and $\Gamma^{\prime}$. Let $g \in G_{k, V_{k}}^{\prime}$ be arbitrary. Then there exists $s \in S_{\Gamma^{\prime}}$ with defect set $V_{k}$ such that $s\left\lceil_{V^{\prime} \backslash V_{k}}=g\right.$. Now, $E^{\prime} \subseteq E$, hence every elementary collapsing of $\Gamma^{\prime}$ is an elementary collapsing of $\Gamma$, as well, Thus $s \in S_{\Gamma}$, and $s$ acts as the identity on $V \backslash V^{\prime}$. Furthermore, if $s^{\prime} \in S_{\Gamma^{\prime}}$ is another element with defect set $V_{k}$ such that $s^{\prime}\left\lceil_{V^{\prime} \backslash V_{k}}=g=s\left\lceil_{V^{\prime} \backslash V_{k}}\right.\right.$, then $s^{\prime} \in S_{\Gamma}$ with $s^{\prime}\left\lceil_{V \backslash V_{k}}=s\left\lceil_{V \backslash V_{k}}\right.\right.$. Thus $\varphi: G_{k, V_{k}}^{\prime} \rightarrow G_{k, V_{k}}, \varphi(g)=s \uparrow_{V \backslash V_{k}}$ is a well defined injective homomorphism of permutation groups.

Second, assume $\left|V^{\prime}\right| \geq k$, and let $V_{k-1}=\left\{v_{1}, \ldots, v_{k-1}\right\} \subseteq V^{\prime}$. Let $v \in V \backslash V^{\prime}$, and let $V_{k}=V_{k-1} \cup\{v\}$. Let $u$ be a neighbor of $v$ and let $e=e_{v u}$. Let $G_{k, V_{k}}$ be the defect $k$-group of $\Gamma$ and let $G_{k-1, V_{k-1}}^{\prime}$ be the defect $(k-1)$-group of $\Gamma^{\prime}$. Let $g \in G_{k-1, V_{k-1}}^{\prime}$ be arbitrary. Then there exists $s \in S_{\Gamma^{\prime}}$ with defect set $V_{k-1}$ such that $s \uparrow_{V^{\prime} \backslash V_{k-1}}=g$. Now, $e s \in S_{\Gamma}$ has defect set $V_{k}$, and $e s \uparrow_{V \backslash V_{k}}$ acts as $g$ on $V^{\prime} \backslash V_{k-1}$, and acts as the identity on $V \backslash\left(V^{\prime} \cup\{v\}\right)$. Furthermore, if $s^{\prime} \in S_{\Gamma^{\prime}}$ is another element with defect set $V_{k-1}$ such that $s^{\prime}\left\lceil_{V^{\prime} \backslash V_{k-1}}=g=s\left\lceil_{V^{\prime} \backslash V_{k-1}}\right.\right.$, then es $\uparrow_{V \backslash V_{k}}=e s^{\prime} \uparrow_{V \backslash V_{k}}$. As $g \in G_{k-1, V_{k-1}}^{\prime}$ was arbitrary, we have that $\varphi: G_{k-1, V_{k-1}}^{\prime} \rightarrow G_{k, V_{k}}, \varphi(g)=e s \uparrow_{V \backslash V_{k}}$ is a well defined injective homomorphism of permutation groups.

Lemma 11. Let $1 \leq m \leq l<k \leq n-2$, and assume $\Gamma$ contains the following subgraph: 


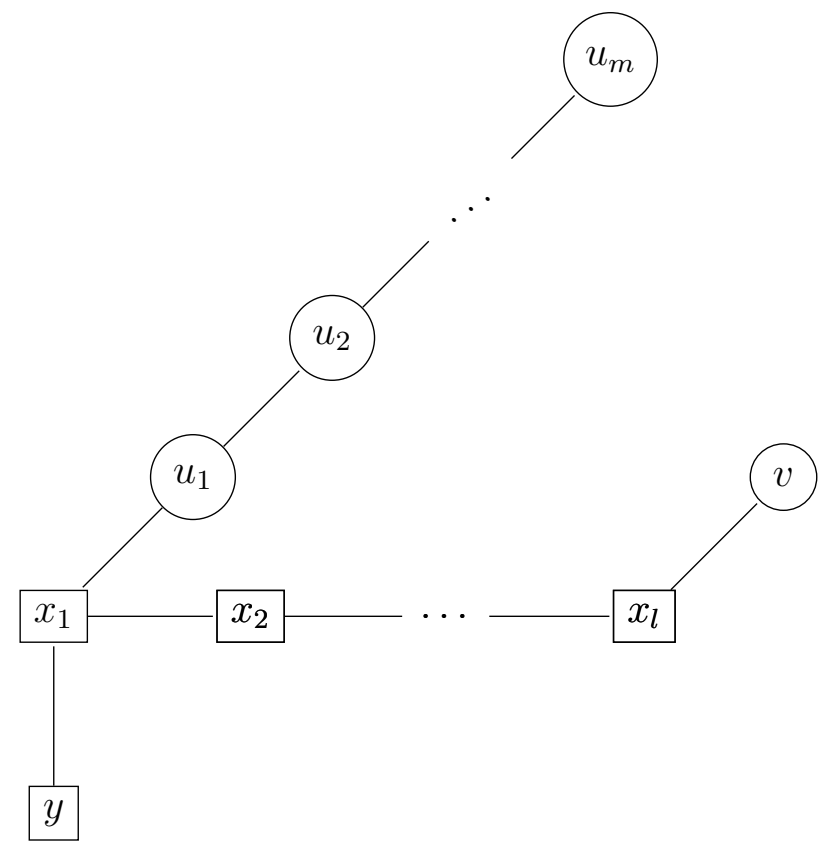

If $V_{k}$ is a set of nodes of size $k$ such that $y, x_{1}, \ldots, x_{l} \in V_{k}$, and $v, u_{i} \notin$ $V_{k}$ for some $1 \leq i \leq m$, then the defect $k$ group $G_{k, V_{k}}$ contains the transposition $\left(u_{i}, v\right)$.

Proof. Let

$$
r= \begin{cases}s s_{1} e_{y x_{1}} e_{x_{1} u_{1}}, & \text { if } i=1 \\ s s_{1} \ldots s_{i} p t t_{i-1} \ldots t_{1} q, & \text { if } i \geq 2\end{cases}
$$

where

$$
\begin{aligned}
s & =e_{v x_{l}} e_{x_{l} x_{l-1}} \ldots e_{x_{2} x_{1}} e_{x_{1} y}, \\
s_{1} & =e_{u_{1} x_{1}} e_{x_{1} x_{2}} \ldots e_{x_{l-1} x_{l}} e_{x_{l} v}, \\
s_{j} & =e_{u_{j} u_{j-1}} \ldots e_{u_{2} u_{1}} e_{u_{1} x_{1}} e_{x_{1} x_{2}} \ldots e_{x_{l-j+1} x_{l-j+2}}, \quad(2 \leq j \leq m), \\
p & =e_{y x_{1}} e_{x_{1} u_{1}} e_{u_{1} u_{2}} \ldots e_{u_{i-1} u_{i}}, \\
t & =e_{x_{l-i+2} x_{l-i+1}} \ldots e_{x_{2} x_{1}} e_{x_{1} y}, \\
t_{j} & =e_{x_{l-j+2} x_{l-j+1}} \ldots e_{x_{2} x_{1}} e_{x_{1} u_{1}} e_{u_{1} u_{2}} \ldots e_{u_{j-1} u_{j}}, \quad(2 \leq j \leq m), \\
t_{1} & =e_{v x_{l}} e_{x_{l} x_{l-1}} \ldots e_{x_{2} x_{1}} e_{x_{1} u_{1}}, \\
q & =e_{y x_{1}} e_{x_{1} x_{2}} \ldots e_{x_{l-1} x_{l}} e_{x_{l} v} .
\end{aligned}
$$

Then $r$ transposes $u_{i}$ and $v$ and fixes all other vertices of $\Gamma$ outside the defect set.

Note that Lemma 11 is going to be useful whenever $\Gamma$ contains a node with degree at least 3 .

Lemma 12. Let $k \geq 2, \Gamma^{\prime}=\left(V^{\prime}, E^{\prime}\right)$ be such that $\left|V^{\prime}\right|>k$ and its defect $k$ group is transitive (e.g. if $\Gamma^{\prime}$ is a cycle with at least $k+1$ vertices). Let $\Gamma=\left(V^{\prime} \cup\{v\}, E^{\prime} \cup\left\{x_{1} v\right\}\right)$ for a new vertex $v$ and 
some $x_{1} \in \Gamma^{\prime}$, where the degree of $x_{1}$ in $\Gamma^{\prime}$ is at least 2. Then the defect $k$ group of $\Gamma$ is isomorphic to $S_{n-k}$.

Proof. Let $n$ be the number of vertices of $\Gamma$, then $n \geq k+2$. Let the vertices of $\Gamma^{\prime}$ be $y, x_{1}, x_{2}, \ldots, x_{k-1}, u_{1}, u_{2}, \ldots, u_{n-k-1}$ such that $u_{1}$ and $y$ are neighbors of $x_{1}$ in $\Gamma^{\prime}$. Let the defect set be $\left\{y, x_{1}, \ldots, x_{k-1}\right\}$. Applying Lemma 11 to the subgraph with vertices $\left\{x_{1}, v, y, u_{1}\right\}$ we obtain that the defect $k$ group of $\Gamma$ contains the transposition $\left(u_{1}, v\right)$. Since the defect $k$ group of $\Gamma^{\prime}$ is transitive and contained in the defect $k$ group of $\Gamma$ by Lemma 10, the defect $k$ group of $\Gamma$ contains the transposition $\left(u_{i}, v\right)$ for all $1 \leq i \leq n-k-1$. Therefore, the defect $k$ group of $\Gamma$ is isomorphic to $S_{n-k}$.

Motivated by Lemma 12, we define the $k$-subgraphs and the maximal $k$-subgraphs of a graph $\Gamma$.

Definition 13. Let $\Gamma$ be a simple connected graph, $k \geq 2$. A connected subgraph $\Gamma^{\prime} \subseteq \Gamma$ is called a $k$-subgraph if its defect $k$ group is the symmetric group of degree $\left|\Gamma^{\prime}\right|-k$. A $k$-subgraph is a maximal $k$ subgraph if it has no proper extension in $\Gamma$ to a $k$-subgraph. Finally, we say that a $k$-subgraph $\Gamma^{\prime}$ is nontrivial if it contains a vertex having at least 3 distinct neighbors in $\Gamma^{\prime}$.

Note that every maximal $k$-subgraph is an induced subgraph. A trivial $k$-subgraph is either a line on $k+1$ points or a cycle on $k+1$ or $k+2$ points. Furthermore, a trivial maximal $k$-subgraph cannot be a cycle by Lemma 12, unless the graph itself is a cycle. Finally, any connected subgraph of $k+1$ points is trivially a $k$-subgraph, thus every connected subgraph of $k+1$ points is contained in a maximal $k$-subgraph. Note that the intersection of two maximal $k$-subgraphs cannot contain more than $k$ vertices:

Lemma 14. Let $\Gamma_{1}, \Gamma_{2}$ be $k$-subgraphs such that $\left|\Gamma_{1} \cap \Gamma_{2}\right|>k$. Then $\Gamma_{1} \cup \Gamma_{2}$ is a k-subgraph, as well.

Proof. Choose the defect set $V_{k}$ such that $V_{k} \varsubsetneqq \Gamma_{1} \cap \Gamma_{2}$, and let $v \in$ $\left(\Gamma_{1} \cap \Gamma_{2}\right) \backslash V_{k}$. Then the symmetric groups acting on $\Gamma_{1} \backslash V_{k}$ and $\Gamma_{2} \backslash V_{k}$ are subgroups in the defect $k$ group of $\Gamma_{1} \cup \Gamma_{2}$. Thus, we can transpose every member of $\Gamma_{i} \backslash\left(V_{k} \cup\{v\}\right)$ with $v$. Therefore, the defect $k$ group of $\Gamma_{1} \cup \Gamma_{2}$ is the symmetric group on $\left(\Gamma_{1} \cup \Gamma_{2}\right) \backslash V_{k}$.

Lemma 15. Let $\Gamma$ be a simple connected graph, and let $\Gamma^{\prime}$ be a $k$ subgraph of $\Gamma$. Let $x_{1} \in \Gamma^{\prime}, v \notin \Gamma^{\prime}$, and let $P=\left(x_{1}, x_{2}, \ldots, x_{l}, v\right)$ be a shortest path between $x_{1}$ and $v$ in $\Gamma$ for some $l \leq k-1$. Assume that $x_{1}$ has at least 2 neighbors in $\Gamma^{\prime}$ apart from $x_{2}$. Then the subgraph $\Gamma^{\prime} \cup P$ is a k-subgraph.

Proof. First, consider the case $x_{2}, \ldots, x_{l} \in \Gamma^{\prime}$. Let $u, y$ be two neighbors of $x_{1}$ in $\Gamma^{\prime}$ distinct from $x_{2}$, and choose the defect set $V_{k}$ such that it 
contains $y, x_{1}, \ldots, x_{l}$ and does not contain $u$. By Lemma 11 the defect $k$ group of $\Gamma^{\prime} \cup\{v\}$ contains the transposition $(u, v)$. Furthermore, the defect $k$ group of $\Gamma^{\prime}$ is the whole symmetric group on $\Gamma^{\prime} \backslash V_{k}$. Thus, the defect $k$ group of $\Gamma^{\prime} \cup\{v\}$ is the whole symmetric group on $\left(\Gamma^{\prime} \backslash V_{k}\right) \cup\{v\}$.

Now, if not all of $x_{2}, \ldots, x_{l}$ are in $\Gamma^{\prime}$, then, by the previous argument, one can add them (and then $v$ ) to $\Gamma^{\prime}$ one by one, and obtain an increasing chain of $k$-subgraphs.

As a corollary, we obtain that every vertex of degree at least 3 together with at least two of its neighbors is contained in exactly one nontrivial maximal $k$-subgraph.

Corollary 16. Let $\Gamma$ be a simple connected graph with $n$ vertices such that $n>k$, and let $x_{1}$ be a vertex having degree at least 3 . Then there exists exactly one maximal $k$-subgraph $\Gamma^{\prime}$ containing $x_{1}$ such that $x_{1}$ has degree at least 2 in $\Gamma^{\prime}$. Furthermore, $\Gamma^{\prime}$ is a nontrivial $k$-subgraph, and if $\Gamma_{x_{1}}$ is the induced subgraph of the vertices in $\Gamma$ that are of at most distance $k-1$ from $x_{1}$, then $\Gamma_{x_{1}} \subseteq \Gamma^{\prime}$.

Proof. Any connected subgraph of $\Gamma$ with $k+1$ vertices containing $x_{1}$ and any two of its neighbors is a $k$-subgraph. Thus there exists at least one maximal $k$-subgraph containing $x_{1}$ and two of its neighbors.

Let $\Gamma^{\prime}$ be a maximal $k$-subgraph containing $x_{1}$ and at least two of its neighbors. Assume that $\Gamma_{x_{1}} \nsubseteq \Gamma^{\prime}$. Let $v \in \Gamma_{x_{1}} \backslash \Gamma^{\prime}$ be any vertex at a minimal distance from $x_{1}$, and let $P=\left(x_{1}, \ldots, x_{l}, v\right)$ be a shortest path between $x_{1}$ and $v$. If $l=1$, then $P=\left(x_{1}, v\right)$. Now $x_{1}$ has at least two neighbors in $\Gamma^{\prime}$ apart from $v$, therefore $\Gamma^{\prime} \cup P$ is a $k$-subgraph by Lemma 15, which contradicts the maximality of $\Gamma^{\prime}$. Thus $l \geq 2$, in particular all neighbors of $x_{1}$ in $\Gamma$ are in $\Gamma^{\prime}$, as well, and thus $\Gamma^{\prime}$ is a nontrivial $k$-subgraph. Hence $x_{1}$ has at least two neighbors in $\Gamma^{\prime}$ apart from $x_{2}$, therefore $\Gamma^{\prime} \cup P$ is a $k$-subgraph by Lemma 15, which contradicts the maximality of $\Gamma^{\prime}$. Thus $\Gamma_{x_{1}} \subseteq \Gamma^{\prime}$.

Now, assume that $\Gamma^{\prime}$ and $\Gamma^{\prime \prime}$ are maximal $k$-subgraphs containing $x_{1}$ and at least two of its neighbors. Then $\Gamma_{x_{1}} \subseteq \Gamma^{\prime}$ and $\Gamma_{x_{1}} \subseteq \Gamma^{\prime \prime}$. Note that either $\Gamma_{x_{1}}=\Gamma$ (and hence $\left|\Gamma_{x_{1}}\right|=n>k$ ), or there exists a vertex $v \in \Gamma$ which is of distance exactly $k$ from $x_{1}$. Let $P=\left(x_{1}, \ldots, x_{k}, v\right)$ be a shortest path between $x_{1}$ and $v$, and let $u$ and $y$ be two neighbors of $x_{1}$ distinct from $x_{2}$. Then $\left\{x_{1}, \ldots, x_{k}, y, u\right\} \subseteq \Gamma_{x_{1}}$, thus $\left|\Gamma_{x_{1}}\right|>k$. Therefore $\left|\Gamma^{\prime} \cap \Gamma^{\prime \prime}\right| \geq\left|\Gamma_{x_{1}}\right|>k$, yielding $\Gamma^{\prime}=\Gamma^{\prime \prime}$ by Lemma 14.

Lemma 17. Let $\Gamma^{\prime}$ be a nontrivial $k$-subgraph of $\Gamma$, and let $P$ be a $\Gamma^{\prime}$-ear. Then $\Gamma^{\prime} \cup P$ is a (nontrivial) k-subgraph of $\Gamma$.

Proof. Let $\Gamma, \Gamma^{\prime}$ and $P=\left(w_{0}, w_{1}, \ldots w_{i}, w_{i+1}\right)$ be a counterexample, where $i$ is minimal. There exists a shortest path $\left(w_{0}, y_{1}, \ldots, y_{l}, w_{i+1}\right)$ in $\Gamma^{\prime}$ among those where the degree of some $y_{j}$ or of $w_{0}$ or of $w_{i+1}$ is at least 3 in $\Gamma^{\prime}$. (At least one such path exists, because $\Gamma^{\prime}$ is connected, 
and is a nontrivial $k$-subgraph, hence contains a vertex of degree at least 3.) For easier notation, let $y_{0}=w_{0}, y_{l+1}=w_{i+1}$. Let $y^{\prime} \in$ $\Gamma^{\prime} \backslash\left\{y_{0}, y_{1}, \ldots, y_{l}, y_{l+1}\right\}$ be a neighbor of $y_{j}$; this exists, because the degree of $y_{j}$ is at least 3 , and otherwise a shorter path would exist between $w_{0}$ and $w_{i+1}$.

If $j+1 \leq k-1$ (that is $j \leq k-2$ ), then by Lemma 15 the induced subgraph on $\Gamma^{\prime} \cup\left\{w_{1}\right\}$ is a $k$-subgraph, thus $\Gamma^{\prime} \cup\left\{w_{1}\right\}$ with the ear $\left(w_{1}, \ldots, w_{i}, w_{i+1}\right)$ is a counterexample with a shorter ear.

Similarly, if $l-j+2 \leq k-1$ (that is $l+3-k \leq j$ ), then by Lemma 15 the induced subgraph on $\Gamma^{\prime} \cup\left\{w_{i}\right\}$ is a $k$-subgraph, thus $\Gamma^{\prime} \cup\left\{w_{i}\right\}$ with the ear $\left(w_{0}, w_{1}, \ldots, w_{i}\right)$ is a counterexample with a shorter ear.

Finally, if $k-1 \leq j \leq l+2-k$, then $2 k-3 \leq l$. Let $\Gamma^{\prime \prime}$ be the cycle $P \cup\left(y_{0}, y_{1}, \ldots, y_{l}, y_{l+1}\right)$ together with $y^{\prime}$ and the edge $y_{j} y^{\prime}$. Then $\Gamma^{\prime \prime}$ is a $k$-subgraph by Lemma 12, $\left|\Gamma^{\prime} \cap \Gamma^{\prime \prime}\right|=l+2 \geq 2 k-1>k$, hence $\Gamma^{\prime} \cup \Gamma^{\prime \prime}=\Gamma^{\prime} \cup P$ is a $k$-subgraph by Lemma 14 .

Corollary 18. Let $\Gamma$ be a simple connected graph with $n$ vertices such that $n>k$, and assume that $\Gamma$ is not a cycle. Suppose uv is an edge contained in a cycle of $\Gamma$. Then there exists exactly one maximal $k$ subgraph $\Gamma^{\prime}$ containing the edge uv. Furthermore, $\Gamma^{\prime}$ is a nontrivial $k$-subgraph, and if $\Gamma_{u v}$ is the 2-edge connected component containing uv, then $\Gamma_{u v} \subseteq \Gamma^{\prime}$.

Proof. Any connected subgraph of $\Gamma$ with $k+1$ vertices containing the edge $u v$ is a $k$-subgraph. Thus there exists at least one maximal $k$-subgraph $\Gamma^{\prime}$ containing the edge $u v$. We prove first that $\Gamma^{\prime}$ is a nontrivial $k$-subgraph, then prove $\Gamma_{u v} \subseteq \Gamma^{\prime}$, and only after that do we prove that $\Gamma^{\prime}$ is unique.

Assume first that $\Gamma^{\prime}$ is a trivial $k$-subgraph. If $\Gamma^{\prime}$ were a cycle, then $\Gamma \backslash \Gamma^{\prime}$ contains at least one vertex, because $\Gamma^{\prime}$ is an induced subgraph of $\Gamma$. Then Lemma 12 contradicts the maximality of $\Gamma^{\prime}$. Thus $\Gamma^{\prime}$ is a line of $k+1$ vertices. Let $\Gamma_{2}$ be a shortest cycle containing $u v$. Now, there must exist a vertex in $\Gamma \backslash \Gamma_{2}$, otherwise either $\Gamma=\Gamma_{2}$ would be a cycle, or there would exist an edge in $\Gamma \backslash \Gamma_{2}$ yielding a shorter cycle than $\Gamma_{2}$ containing the edge $u v$. Let $x_{2} \in \Gamma \backslash \Gamma_{2}$ be a neighbor of a vertex in $\Gamma_{2}$. By Lemma 12 the induced subgraph on $\Gamma_{2} \cup\left\{x_{2}\right\}$ is a $k$-subgraph. Thus $\Gamma^{\prime} \nsubseteq \Gamma_{2}$, otherwise $\Gamma^{\prime}$ would not be a maximal $k$-subgraph. Let $x_{1} \in \Gamma^{\prime} \cap \Gamma_{2}$ be a vertex such that two of its neighbors are in $\Gamma_{2}$ and its third neighbor is some $x_{2} \in \Gamma^{\prime} \backslash \Gamma_{2}$. Note that every vertex in $\Gamma^{\prime}$ is of distance at most $k-1$ from $x_{1}$, because $u, v \in \Gamma^{\prime} \cap \Gamma_{2}$. Thus, if $\left|\Gamma_{2}\right| \geq k+1$, then $\Gamma_{2}$ together with $x_{2}$ and the edge $x_{1} x_{2}$ is a $k$-subgraph by Lemma 12, and hence $\Gamma_{2} \cup \Gamma^{\prime}$ is a $k$-subgraph by Lemma 15, contradicting the maximality of $\Gamma^{\prime}$. Otherwise, if $\left|\Gamma_{2}\right| \leq k$, then every vertex in $\Gamma_{2}$ is of distance at most $k-1$ from $x_{1}$, and hence $\Gamma_{2} \cup \Gamma^{\prime}$ is a $k$-subgraph by Lemma 15, contradicting the maximality of $\Gamma^{\prime}$. Therefore $\Gamma^{\prime}$ is a nontrivial $k$-subgraph. 
Now we show that the two-edge connected component $\Gamma_{u v} \subseteq \Gamma^{\prime}$. Let $\Gamma, \Gamma^{\prime}$ be a counterexample to this such that the number of vertices of $\Gamma_{u v}$ is minimal, and among these counterexamples choose one where the number of edges of $\Gamma_{u v}$ is minimal. Using an ear-decomposition [11, $\Gamma_{u v}$ is either a cycle, or there exists a 2-edge connected subgraph $\Gamma_{1} \subseteq \Gamma_{u v}$ and there exists

(1) either a $\Gamma_{1}$-ear $P$ such that $\Gamma_{u v}=\Gamma_{1} \cup P$,

(2) or a cycle $\Gamma_{2}$ such that $\left|\Gamma_{1} \cap \Gamma_{2}\right|=1$ and $\Gamma_{u v}=\Gamma_{1} \cup \Gamma_{2}$.

If $\Gamma_{u v}$ is a cycle containing the edge $u v$, and $\Gamma_{u v} \nsubseteq \Gamma^{\prime}$, then going along the edges of $\Gamma_{u v}$, one can find a $\Gamma^{\prime}$-ear $P \subseteq \Gamma_{u v}$. Then $\Gamma^{\prime} \cup P$ is a $k$-subgraph by Lemma 17, contradicting the maximality of $\Gamma^{\prime}$. Thus $\Gamma_{u v}$ is not a cycle. Let us choose $\Gamma_{1}$ from cases (11) and (2) so that it would have the least number of vertices.

Assume first that case (1) holds. By minimality of the counterexample, $\Gamma_{1} \subseteq \Gamma^{\prime}$. If $P \nsubseteq \Gamma^{\prime}$, then going along the edges of $P$ one can find a $\Gamma^{\prime}$-ear $P^{\prime} \subseteq P$. But then $\Gamma^{\prime} \cup P^{\prime}$ is a $k$-subgraph by Lemma 17 , contradicting the maximality of $\Gamma^{\prime}$.

Assume now that case (2) holds. Again, by induction, $\Gamma_{1} \subseteq \Gamma^{\prime}$. If $\Gamma_{2} \not \Gamma^{\prime}$, then either $\left|\Gamma^{\prime} \cap \Gamma_{2}\right|=1$ or going along the edges of $\Gamma_{2}$ one can find a $\Gamma^{\prime}$-ear $P^{\prime} \subseteq \Gamma_{2}$. The latter case cannot happen, because then $\Gamma^{\prime} \cup P^{\prime}$ is a $k$-subgraph by Lemma 17, contradicting the maximality of $\Gamma^{\prime}$. Thus $\left|\Gamma^{\prime} \cap \Gamma_{2}\right|=1$, and hence $\Gamma^{\prime} \cap \Gamma_{2}=\Gamma_{1} \cap \Gamma_{2}$. Let $\Gamma_{1} \cap \Gamma_{2}=\left\{x_{1}\right\}$, and let $v_{1}$ be a neighbor of $x_{1}$ in $\Gamma_{1} \backslash \Gamma_{2}$, and let $v_{2}$ be a neighbor of $x_{1}$ in $\Gamma_{2} \backslash \Gamma_{1}$. If $\left|\Gamma_{2}\right| \leq k$, then $\Gamma_{2}$ can be extended to a connected subgraph of $\Gamma$ having exactly $k+1$ vertices, which is a $k$-subgraph. If $\left|\Gamma_{2}\right| \geq k+1$, then $\Gamma_{2} \cup\left\{v_{1}\right\}$ is a $k$-subgraph by Lemma 12, In any case, there exists a maximal $k$-subgraph $\Gamma_{2}^{\prime} \supseteq \Gamma_{2}$. For notational convenience, let $\Gamma_{1}^{\prime}$ denote the maximal $k$-subgraph $\Gamma^{\prime}$ containing $\Gamma_{1}$. We prove that $\Gamma_{2}^{\prime}=\Gamma_{1}^{\prime}=\Gamma^{\prime}$, thus $\Gamma^{\prime}$ contains $\Gamma_{2}$, contradicting that we chose a counterexample.

Now, both $\Gamma_{1}$ and $\Gamma_{2}$ contain at least two neighbors of $x_{1}$. Let $V_{i} \subseteq \Gamma_{i}$ be the set of vertices with distance at most $k-1$ from $x_{1}(i \in\{1,2\})$. If $\left|\Gamma_{i}\right| \leq k$, then $V_{i}$ contains all vertices of $\Gamma_{i}$, otherwise $\left|V_{i}\right| \geq k$ $(i \in\{1,2\})$. By Lemma 15, the induced subgraph on $V_{1}$ is contained in $\Gamma_{2}^{\prime}$. Thus, if $V_{1}$ contains all vertices of $\Gamma_{1}$, then $\Gamma_{1} \subseteq \Gamma_{2}^{\prime}$, hence we have $\Gamma_{1}^{\prime}=\Gamma_{2}^{\prime}$. Similarly, the induced subgraph on $V_{2}$ is contained in $\Gamma_{1}^{\prime}$. Thus, if $V_{2}$ contains all vertices of $\Gamma_{2}$, then $\Gamma_{2} \subseteq \Gamma_{1}^{\prime}$, hence we have $\Gamma_{1}^{\prime}=\Gamma_{2}^{\prime}$. Otherwise, $\left|\Gamma_{1}^{\prime} \cap \Gamma_{2}^{\prime}\right| \geq\left|V_{1}\right|+\left|V_{2}\right|-\left|\left\{x_{1}\right\}\right| \geq 2 k-1>k$, hence by Lemma 14 we have $\Gamma_{1}^{\prime}=\Gamma_{2}^{\prime}$.

Finally, we prove uniqueness. Let $\Gamma^{\prime}$ and $\Gamma^{\prime \prime}$ be two maximal $k$ subgraphs containing the edge $u v$. Then both $\Gamma^{\prime}$ and $\Gamma^{\prime \prime}$ contain $\Gamma_{u v}$. If $\Gamma=\Gamma_{u v}$, then $\Gamma^{\prime}=\Gamma_{u v}=\Gamma^{\prime \prime}$. Otherwise, there exists a vertex $x_{2} \in$ $\Gamma \backslash \Gamma_{u v}$ such that it has a neighbor $x_{1} \in \Gamma_{u v}$. Note that $x_{1}$ has degree at least 3 in $\Gamma$. Let $V_{1}$ be the vertices of $\Gamma$ of distance at most $k-1$ from $x_{1}$. Note that if $V_{1}$ does not contain all vertices of $\Gamma$, then $\left|V_{1}\right|>k$. 
By 2-edge connectivity, $\Gamma_{u v} \subseteq \Gamma^{\prime}$ contains at least two neighbors of $x_{1}$, thus $V_{1} \subseteq \Gamma^{\prime}$ by Lemma 15. Similarly, $\Gamma_{u v} \subseteq \Gamma^{\prime \prime}$ contains at least two neighbors of $x_{1}$, thus $V_{1} \subseteq \Gamma^{\prime \prime}$ by Lemma 15. If $V_{1}$ contains all vertices of $\Gamma$, then $\Gamma^{\prime}=\Gamma=\Gamma^{\prime \prime}$. Otherwise, $\left|\Gamma^{\prime} \cap \Gamma^{\prime \prime}\right| \geq\left|V_{1}\right|>k$, and $\Gamma^{\prime}=\Gamma^{\prime \prime}$ by Lemma 14 .

Recall that by [11] a strongly connected antisymmetric digraph becomes a 2-edge connected graph after forgetting the directions. Thus Rhodes's conjecture about strongly connected, antisymmetric digraphs [9, Conjecture 6.51i (3)-(4)] follows immediately from the following theorem on 2-edge connected graphs:

Theorem 19. Let $n>k \geq 2$, $\Gamma$ be a 2-edge connected simple graph having $n$ vertices. If $\Gamma$ is a cycle, then the defect $k$ group is $Z_{n-k}$. If $\Gamma$ is not a cycle, then the defect $k$ group is $S_{n-k}$.

Proof. If $\Gamma$ is a cycle, then its defect $k$ group is $Z_{n-k}$ by Lemma 8 . Since $\Gamma$ is 2-edge connected with at least 3 vertices, every edge of $\Gamma$ is contained in a cycle. Thus, if $\Gamma$ is not a cycle, then the defect $k$ group is $S_{n-k}$ by Corollary 18 ,

The final part of this section is devoted to prove item (5b) of Theorem 1, First, we define bridges in $\Gamma$ :

Definition 20. A path $\left(x_{1}, \ldots, x_{l}\right)$ in a connected graph $\Gamma$ for some $l \geq 2$ is called a bridge if the degree of $x_{i}$ in $\Gamma$ is 2 for all $2 \leq i \leq l-1$, and if $\Gamma \backslash\left\{x_{j} x_{j+1}\right\}$ is disconnected for all $1 \leq j \leq l-1$. The length of the bridge $\left(x_{1}, \ldots, x_{l}\right)$ is $l$.

The intersection of maximal $k$-subgraphs turn out to be bridges:

Lemma 21. Let $\Gamma_{1}$ and $\Gamma_{2}$ be distinct maximal $k$-subgraphs of the connected simple graph $\Gamma$. Assume that $\Gamma$ is not a cycle. Then $\Gamma_{1} \cap \Gamma_{2}$ is either empty, or is a bridge $\left(x_{1}, \ldots, x_{l}\right)$ such that

(1) $l \leq k$, and

(2) if $l \geq 2$ and $\Gamma_{i} \backslash\left\{x_{1}, \ldots, x_{l}\right\}$ ( $\left.i \in\{1,2\}\right)$ contains a neighbor of $x_{1}$ (resp. $\left.x_{l}\right)$, then $\Gamma_{i}$ contains all neighbors of $x_{1}$ (resp. $x_{l}$ ),

Proof. Note that $\Gamma_{1}$ and $\Gamma_{2}$ are induced subgraphs of $\Gamma$, thus so is $\Gamma_{1} \cap \Gamma_{2}$.

We prove first that $\Gamma_{1} \cap \Gamma_{2}$ is connected (or empty) if $\Gamma_{1}$ is a nontrivial maximal $k$-subgraph. Suppose that $u, v \in \Gamma_{1} \cap \Gamma_{2}$ are in different components of $\Gamma_{1} \cap \Gamma_{2}$ such that the distance between $u$ and $v$ is minimal in $\Gamma_{2}$. Due to the minimality, there exists a path $\left(u, x_{1}, \ldots, x_{l}, v\right)$ such that $x_{1}, \ldots, x_{l} \in \Gamma_{2} \backslash \Gamma_{1}$. Then $P=\left(u, x_{1}, \ldots, x_{l}, v\right)$ is a $\Gamma_{1}$-ear, and $\Gamma_{1} \cup P$ would be a $k$-subgraph by Lemma 17, contradicting the maximality of $\Gamma_{1}$. Thus $\Gamma_{1} \cap \Gamma_{2}$ is connected. One can prove similarly that $\Gamma_{1} \cap \Gamma_{2}$ is connected if $\Gamma_{2}$ is a nontrivial maximal $k$-subgraph.

Now we prove that $\Gamma_{1} \cap \Gamma_{2}$ is connected, even if both $\Gamma_{1}$ and $\Gamma_{2}$ are trivial maximal $k$-subgraphs. As $\Gamma_{1} \varsubsetneqq \Gamma, \Gamma_{1}$ cannot be a cycle 
hence must be a line $\left(x_{1}, \ldots, x_{k+1}\right)$. Note that the degree of $x_{i}$ in $\Gamma$ for $2 \leq i \leq k$ must be 2 , otherwise a nontrivial maximal $k$-subgraph would contain $x_{i}$, and thus also $\Gamma_{1}$ by Corollary 16. In particular, if $\Gamma_{1} \cap \Gamma_{2}$ is not connected, then $x_{1}, x_{k+1} \in \Gamma_{1} \cap \Gamma_{2}, x_{i} \notin \Gamma_{1} \cap \Gamma_{2}$ for some $2 \leq i \leq k$, and $\Gamma_{1} \cup \Gamma_{2}$ would be a cycle. However, by Corollary 18, the edge $x_{1} x_{2}$ is contained in a unique nontrivial maximal $k$-subgraph, contradicting that it is also contained in the trivial maximal $k$-subgraph $\Gamma_{1}$.

Now, we prove (11). By Corollary 18, $\Gamma_{1} \cap \Gamma_{2}$ cannot contain any edge $u v$ which is contained in a cycle. As $\Gamma_{1} \cap \Gamma_{2}$ is connected, it must be a tree. However, $\Gamma_{1} \cap \Gamma_{2}$ cannot contain any vertex of degree at least 3 in $\Gamma_{1} \cap \Gamma_{2}$, otherwise that vertex would be contained in a unique maximal $k$-subgraph by Corollary 16. Thus $\Gamma_{1} \cap \Gamma_{2}$ is a path $\left(x_{1}, \ldots, x_{l}\right)$. Now, $l \leq k$ by Lemma 14, proving (11). Note that if any $x_{i}(2 \leq i \leq l-1)$ is of degree at least 3 in $\Gamma$, then $\left\{x_{i-1}, x_{i}, x_{i+1}\right\}$ is contained in a unique maximal $k$-subgraph by Corollary 16, a contradiction. For (2) observe that at least two neighbors of $x_{1}\left(\operatorname{resp} . x_{l}\right)$ are in $\Gamma_{i}$, and thus all its neighbors must be in $\Gamma_{i}$ by Corollary 16. Finally, if $l \geq 2$ then $\Gamma \backslash\left\{x_{j} x_{j+1}\right\}$ is disconnected for all $1 \leq j \leq l-1$ follows immediately from Corollary 18 and the fact that any edge that is not contained in any cycle disconnects the graph $\Gamma$.

Edges of short maximal bridges (having length at most $k-1$ ) are contained in a unique maximal $k$-subgraph:

Lemma 22. Let $\Gamma$ be a simple connected graph with $n$ vertices such that $n>k$, and let $u v$ be an edge which is not contained in any cycle. Let $\left(x_{1}, \ldots, x_{l}\right)$ be a longest bridge containing the edge uv. If $l \leq k-1$, then $u v$ is contained in a unique maximal $k$-subgraph $\Gamma^{\prime}$, and furthermore, $\Gamma^{\prime}$ is a nontrivial k-subgraph.

Proof. As $u v$ is not part of any cycle in $\Gamma$, uv is a bridge of length 2 . Note that a longest bridge $\left(x_{1}, \ldots, x_{l}\right)$ containing $u v$ is unique, because as long as the degree of at least one of the path's end vertices is 2 in $\Gamma$, the path can be extended in that direction. The obtained path is the unique longest bridge containing $u v$.

Let $\Gamma^{\prime}$ be a maximal $k$-subgraph containing $u v$, and assume $l \leq k-1$. Note that the distance of $x_{1}$ and $x_{l}$ is $l-1 \leq k-2$. As $|\Gamma| \geq k+1$, at least one of $x_{1}$ and $x_{l}$ has degree at least 3 in $\Gamma$, say $x_{1}$. We distinguish two cases according to the degree of $x_{l}$.

Assume first that $x_{l}$ is of degree 1 . As $\Gamma^{\prime}$ is a connected subgraph having at least $k+1$ vertices, $\Gamma^{\prime}$ must contain $x_{1}$ and at least two of its neighbors. Then by Corollary 16 it contains all vertices of $\Gamma$ of distance at most $k-1$ from $x_{1}$. In particular, $\Gamma^{\prime}$ must contain the bridge $\left(x_{1}, \ldots, x_{l}\right)$. However, there is a unique (nontrivial) maximal $k$-subgraph $\Gamma_{1}^{\prime}$ containing $x_{1}$ and two of its neighbors by Corollary 16, and thus $\Gamma^{\prime}=\Gamma_{1}^{\prime}$ is that unique maximal $k$-subgraph. 
Assume now that $x_{l}$ is of degree at least 3. As $\Gamma^{\prime}$ is a connected subgraph having at least $k+1$ vertices, $\Gamma^{\prime}$ must contain $x_{1}$ and at least two of its neighbors, or $x_{l}$ and at least two of its neighbors. If $\Gamma^{\prime}$ contains $x_{1}$ and at least two of its neighbors, then by Corollary 16] it contains all vertices of $\Gamma$ of distance at most $k-1$ from $x_{1}$. In particular, $\Gamma^{\prime}$ must contain the bridge $\left(x_{1}, \ldots, x_{l}\right)$ and all of the neighbors of $x_{l}$. Similarly, one can prove that if $\Gamma^{\prime}$ contains $x_{l}$ and two of its neighbors, then it also contains the bridge $\left(x_{1}, \ldots, x_{l}\right)$ and all of the neighbors of $x_{1}$. However, there is a unique (nontrivial) maximal $k$-subgraph $\Gamma_{1}^{\prime}$ containing $x_{1}$ and two of its neighbors by Corollary 16, and also a unique (nontrivial) maximal $k$-subgraph $\Gamma_{l}^{\prime}$ containing $x_{l}$ and two of its neighbors by Corollary 16. Therefore $\Gamma^{\prime}$ must equal to both $\Gamma_{1}^{\prime}$ and $\Gamma_{l}^{\prime}$, and hence is unique.

In particular, in non-cycle graphs trivial maximal $k$-subgraphs or intersections of two different maximal $k$-subgraphs consist of edges that are contained in long bridges (having length at least $k$ ). The key observation in proving item (5b) of Theorem 1 is that a defect $k$ group cannot move a vertex across a bridge of length at least $k$ :

Lemma 23. Let $2 \leq k \leq l, \Gamma_{1}$ and $\Gamma_{2}$ be disjoint connected subgraphs of the connected graph $\Gamma$, and $\left(x_{1}, x_{2}, \ldots, x_{l}\right)$ be a bridge in $\Gamma$ such that $x_{1} \ldots, x_{l} \notin \Gamma_{1} \cup \Gamma_{2}, x_{1}$ has only neighbors in $\Gamma_{1}$ (except for $x_{2}$ ), $x_{l}$ has only neighbors in $\Gamma_{2}$ (except for $x_{l-1}$ ). Assume $\Gamma$ has no more vertices than $\Gamma_{1} \cup \Gamma_{2} \cup\left(x_{1}, \ldots, x_{l}\right)$. Let the defect set be $V_{k}=\left\{x_{1}, \ldots, x_{k}\right\}$. Then for any $u \in \Gamma_{1}$ and $v \in \Gamma_{2}$ there does not exist any permutation in $G_{k, V_{k}}$ which moves $u$ to $v$.

Proof. Let $S=S_{\Gamma}$. Assume that there exists $u \in \Gamma_{1}, v \in \Gamma_{2}$, and a transformation $g \in S$ of defect $V_{k}$ such that $g\left\lceil_{V \backslash V_{k}} \in G_{k, V_{k}}\right.$ and $u g=v$. Let $s_{0} \in G_{k, V_{k}}$ be the unique idempotent power of $g$, that is $s_{0}$ is a transformation of defect $V_{k}$ that acts as the identity on $\Gamma \backslash V_{k}$. Then there exists a series of elementary collapsings $e_{1}, \ldots, e_{m}$ such that $g=e_{1} \ldots e_{m}$. For every $1 \leq d \leq m$ let $s_{d}=s_{0} e_{1} \ldots e_{d}$. Now, $s_{m}=s_{0} e_{1} \ldots e_{m}=s_{0} g=g s_{0}=g$. In particular, both $s_{m}$ and $s_{0}$ are of defect $k$, hence $s_{d}$ is of defect $k$ for all $1 \leq d \leq m$. Consequently, $\left|\Gamma_{1} s_{d}\right|=\left|\Gamma_{1}\right|,\left|\Gamma_{2} s_{d}\right|=\left|\Gamma_{2}\right|$ and $\Gamma_{1} s_{d} \cap \Gamma_{2} s_{d}=\emptyset$ for all $1 \leq d \leq m$.

For an arbitrary $s \in S$, let

$$
i(s)= \begin{cases}0, & \text { if } \Gamma_{1} s \subseteq \Gamma_{1}, \\ l+1, & \text { if } \Gamma_{1} s \nsubseteq \Gamma_{1} \cup\left\{x_{1}, \ldots, x_{l}\right\}, \\ \min _{1 \leq i \leq l}\left\{\Gamma_{1} s \subseteq \Gamma_{1} \cup\left\{x_{1}, \ldots, x_{i}\right\}\right\}, & \text { otherwise. }\end{cases}
$$


Similarly, let

$j(s)= \begin{cases}l+1, & \text { if } \Gamma_{2} s \subseteq \Gamma_{2}, \\ 0, & \text { if } \Gamma_{2} s \nsubseteq \Gamma_{2} \cup\left\{x_{1}, \ldots, x_{l}\right\}, \\ \max _{1 \leq j \leq l}\left\{\Gamma_{2} s \subseteq \Gamma_{2} \cup\left\{x_{j}, \ldots, x_{l}\right\}\right\}, & \text { otherwise. }\end{cases}$

Note that for arbitrary $s \in S$ and elementary collapsing $e$, we have $|i(s)-i(s e)| \leq 1,|j(s)-j(s e)| \leq 1$. Furthermore, both $\left|i\left(s_{d}\right)-i\left(s_{d} e\right)\right|=$ 1 and $\left|j\left(s_{d}\right)-j\left(s_{d} e\right)\right|=1$ cannot happen at the same time for any $1 \leq d \leq m$, because that would contradict $\Gamma_{1} s_{d} \cap \Gamma_{2} s_{d} \neq \emptyset$.

For $s_{0}$ we have $i\left(s_{0}\right)=0<l+1=j\left(s_{0}\right)$, for $s_{m}$ we have $i\left(s_{m}\right)=$ $l+1 \geq j\left(s_{m}\right)$. Let $1 \leq d \leq m$ be minimal such that $i\left(s_{d}\right) \geq j\left(s_{d}\right)$. Then $i\left(s_{d-1}\right)<j\left(s_{d-1}\right)$. From $s_{d-1}$ to $s_{d}$ either $i$ or $j$ can change and by at most 1 , thus $i\left(s_{d}\right)=j\left(s_{d}\right)$. If $i\left(s_{d}\right)=j\left(s_{d}\right) \in\{1, \ldots, l\}$, then $x_{i\left(s_{d}\right)} \in \Gamma_{1} s_{d} \cap \Gamma_{2} s_{d}$, contradicting $\Gamma_{1} s_{d} \cap \Gamma_{2} s_{d}=\emptyset$. Thus $i\left(s_{d}\right)=j\left(s_{d}\right) \notin$ $\{1, \ldots, l\}$. Assume $i\left(s_{d}\right)=j\left(s_{d}\right)=l+1$, the case $i\left(s_{d}\right)=j\left(s_{d}\right)=0$ can be handled similarly.

Now, $j\left(s_{d}\right)=l+1$ yields $\Gamma_{2} s_{d} \subseteq \Gamma_{2}$. Furthermore, $\left|\Gamma_{2} s_{d}\right|=\left|\Gamma_{2}\right|$, thus $\Gamma_{2} s_{d}=\Gamma_{2}$. From $i\left(s_{d}\right)=l+1$ we have $\Gamma_{1} s_{d} \cap \Gamma_{2} \neq \emptyset$. Thus $\Gamma_{1} s_{d} \cap \Gamma_{2} s_{d}=\Gamma_{1} s_{d} \cap \Gamma_{2} \neq \emptyset$, a contradiction.

Corollary 24. Let $\Gamma_{1}$ and $\Gamma_{2}$ be connected subgraphs of $\Gamma$ such that $\Gamma_{1} \cap \Gamma_{2}$ is a length $k$ bridge in $\Gamma$. Let $V_{k}=\Gamma_{1} \cap \Gamma_{2}$ be the defect set. Let $G_{i}$ be the defect $k$ group of $\Gamma_{i}, G$ be the defect $k$ group of $\Gamma_{1} \cup \Gamma_{2}$. Then

$$
G=G_{1} \times G_{2} .
$$

Proof. By Lemma 10 we have $G_{1}, G_{2} \leq G$. Since $G_{1}$ and $G_{2}$ act on disjoint vertices, their elements commute. Thus $G_{1} \times G_{2} \leq G$. Now, $V_{k}$ is a bridge of length $k$, thus by Lemma 23 (applied to the disjoint subgraphs $\Gamma_{1} \backslash V_{k}$ and $\Gamma_{2} \backslash V_{k}$ ) there exists no element of $G$ moving a vertex from $\Gamma_{1}$ to $\Gamma_{2}$ or vice versa. Therefore $G \leq G_{1} \times G_{2}$.

Finally, we are ready to prove item (5b) of Theorem 1 .

Proof of item (5b) of Theorem 11. If $\Gamma$ is a cycle, then its defect $k$ group is $Z_{n-k}$ by Lemma 8. Otherwise, we prove the theorem by induction on the number of maximal $k$-subgraphs of $\Gamma$. If $\Gamma$ is a maximal $k$-subgraph, then the theorem holds, and the defect $k$ group of $\Gamma$ is $S_{n-k}$. In the following we assume that $\Gamma$ contains $m$-many maximal $k$-subgraphs for some $m \geq 2$, and that the theorem holds for all graphs with at most $(m-1)$-many maximal $k$-subgraphs.

We consider two cases. Assume first that there exists a degree 1 vertex $x_{1} \in \Gamma$, such that there exists a path $\left(x_{1}, \ldots, x_{k+1}\right)$ which is a bridge. Let $\Gamma_{1}$ be the path $\left(x_{1}, \ldots, x_{k+1}\right)$, and let $\Gamma_{2}$ be $\Gamma \backslash\left\{x_{1}\right\}$. Now, $\Gamma_{1}$ is a trivial maximal $k$-subgraph, hence $\Gamma_{2}$ contains the same maximal $k$-subgraphs as $\Gamma$ except $\Gamma_{1}$. Furthermore, $\Gamma_{2}$ is connected, 
and cannot be a cycle because the degree of $x_{2}$ in $\Gamma_{2}$ is 1 . Let the sizes of the maximal $k$-subgraphs of $\Gamma_{2}$ be $n_{2}, \ldots, n_{m}$, then by induction the defect $k$ group of $\Gamma_{1}$ is $S_{n_{2}-k} \times \cdots \times S_{n_{m}-k}$. The size of $\Gamma_{1}$ is $n_{1}=k+1$, its defect $k$-group is $S_{n_{1}-k}$. Furthermore, $\Gamma_{1} \cap \Gamma_{2}$ is a bridge of length $k$. By Corollary 24 the defect $k$-group of $\Gamma$ is $S_{n_{1}-k} \times S_{n_{2}-k} \times \cdots \times S_{n_{m}-k}$.

In the second case, no degree 1 vertex $x_{1}$ is in a path $\left(x_{1}, \ldots, x_{k+1}\right)$ which is a bridge. Then any maximal bridge $\left(x_{1}, \ldots, x_{l}\right)$ with a degree 1 vertex $x_{1}$ has length $l \leq k$, and, as the bridge cannot be extended, $x_{l}$ must have degree at least 3 . Moreover, $\left(x_{1}, \ldots, x_{l}\right)$ lies in a maximal $k$-subgraph containing $x_{l}$ and all its neighbors by Lemma 22 and Corollary 16. In particular every bridge in $\Gamma$ of length at least $k+1$ occurs between nodes of degree at least 3 . Hence every bridge of length at least $k+1$ occurs between two nontrivial maximal $k$-subgraphs by Corollary 16. For every vertex $v$ having degree at least 3 in $\Gamma$, let $\Gamma_{v}$ be the unique maximal $k$-subgraph containing $v$ and all its neighbors (Corollary 16). By definition, these are all the nontrivial maximal $k$-subgraphs of $\Gamma$.

Let $\Gamma^{k}$ be the graph whose vertices are the nontrivial maximal $k$ subgraphs, and $\Gamma_{u} \Gamma_{v}$ is an edge in $\Gamma^{k}$ (for $\Gamma_{u} \neq \Gamma_{v}$ ) if and only if there exists a bridge in $\Gamma$ between a vertex $u^{\prime} \in \Gamma_{u}$ of degree at least 3 in $\Gamma_{u}$ and a vertex $v^{\prime} \in \Gamma_{v}$ of degree at least 3 in $\Gamma_{v}$. By Corollary 18, $\Gamma_{u}=\Gamma_{v}$ if $u$ and $v$ are in the same 2-edge connected component. As the 2-edge connected components of $\Gamma$ form a tree, the graph $\Gamma^{k}$ is a tree.

Now, $\Gamma^{k}$ has $m$ vertices. Let $\Gamma_{1}$ be a leaf in $\Gamma^{k}$, and let $\Gamma_{m}$ be its unique neighbor in $\Gamma^{k}$. Let $x_{1} \in \Gamma_{1}$ and $x_{l} \in \Gamma_{m}$ be the unique vertices of degree at least 3 in $\Gamma_{i}(i \in\{1, l\})$ such that there exists a bridge $P=\left(x_{1}, \ldots, x_{l}\right)$ in $\Gamma$. Note that the length of $P$ is at least $k$, otherwise $\Gamma_{1}=\Gamma_{m}$ would follow by Lemma 22 . Furthermore, any other bridge having an endpoint in $\Gamma_{1}$ must be of length at most $k$, because every degree 1 vertex is of distance at most $k-1$ from a vertex of degree at least 3. Thus every bridge other than $P$ and having an endpoint in $\Gamma_{1}$ is a subset of $\Gamma_{1}$ by Corollary 16.

Let $\Gamma_{2}=\left(\Gamma \backslash \Gamma_{1}\right) \cup P$. Now, $\Gamma_{1}$ is a maximal $k$-subgraph, $\Gamma_{2}$ has one less maximal $k$-subgraphs than $\Gamma$. Furthermore, $\Gamma_{2}$ is connected, because every bridge other than $P$ and having an endpoint in $\Gamma_{1}$ is a subset of $\Gamma_{1}$. Finally, $\Gamma_{2}$ is not a cycle, because it contains the vertex $x_{1}$ which is of degree 1 in $\Gamma_{2}$. Let the sizes of the maximal $k$-subgraphs of $\Gamma_{2}$ be $n_{2}, \ldots, n_{m}$, then by induction the defect $k$ group of $\Gamma_{1}$ is $S_{n_{2}-k} \times \cdots \times S_{n_{m}-k}$. Let the size of $\Gamma_{1}$ be $n_{1}$, its defect $k$-group is $S_{n_{1}-k}$. Furthermore, $\Gamma_{1} \cap \Gamma_{2}$ is a bridge of length $k$. By Corollary 24 the defect $k$-group of $\Gamma$ is $S_{n_{1}-k} \times S_{n_{2}-k} \times \cdots \times S_{n_{m}-k}$. 


\section{An Algorithm to CAlCUlate the DefeCt $k$ GROUP}

Note that by items (3) and (4) of Theorem 11 the defect 1 group can be trivially computed in $O(|E|)$ time by first determining the 2-vertex connected components [8], and whether each is a cycle, the exceptional graph (Figure 1) or if not, whether or not it is bipartite.

For $k \geq 2$ one can check first if $\Gamma$ is a cycle (and then the defect group is $Z_{n-k}$ ) or a path (and then the defect group is trivial). In the following, we give a linear algorithm (running in $O(|E|)$ time) to determine the maximal $k$-subgraphs $(k \geq 2)$ of a connected graph $\Gamma$ having $n$ vertices, $|E|$ edges where at least one vertex is of degree at least 3 .

During the algorithm we color the vertices. Let us call a maximal subgraph with vertices having the same color a monochromatic component. First, one finds all 2-edge connected components and the tree of two-edge connected components in $O(|E|)$ time using e.g. [13]. Color the vertices of the nontrivial (i.e. having size greater than 1) 2-edge connected components such that two distinct vertices have the same color if and only if they are in the same nontrivial 2-edge connected component. Furthermore, color the uncolored vertices having degree at least 3 by different colors from each other and from the colors of the 2-edge connected components. Then the monochromatic components are each contained in a unique nontrivial maximal $k$-subgraph by Corollaries 16] and 18 (a nontrivial maximal $k$-subgraph may contain more than one of these monochromatic components). Furthermore, the monochromatic components and the degree 1 vertices are connected by bridges. If any of the bridges connecting two monochromatic components is of length at most $k-1$, then recolor the two monochromatic components at the ends of the bridge and the vertices of the bridge by the same color, because these are contained in the same maximal $k$-subgraph by Corollary 16. Similarly, if any of the bridges connecting a monochromatic component and a degree 1 vertex is of length at most $k-1$, then recolor the monochromatic component and the vertices of the bridge by the same color, because these are contained in the same maximal $k$-subgraph by Lemma 22, Repeat recoloring along all bridges of length at most $k-1$ in $O(|E|)$ time. Then we obtain monochromatic components $\Gamma_{1}, \ldots, \Gamma_{l}$ connected by long bridges (i.e. bridges of length at least $k$ ), and possibly some long bridges to degree 1 vertices. Now, we have finished coloring.

For every $1 \leq i \leq l$, let $\Gamma_{i}^{\prime}$ be the induced subgraph having all vertices of distance at most $k-1$ from $\Gamma_{i}$, which can be obtained in $O(|E|)$ time by adding the appropriate $k-1$ vertices of the long bridges to the appropriate monochromatic component. Note that the obtained induced subgraphs are not necessarily disjoint. Then $\Gamma_{1}^{\prime}, \ldots, \Gamma_{l}^{\prime}$ are the nontrivial maximal $k$-subgraphs of $\Gamma$ by Lemma 22, Again, by Lemma 22, 
the trivial maximal $k$-subgraphs of $\Gamma$ are the paths containing exactly $k+1$ vertices in a long bridge. These can also be computed in $O(|E|)$ time by going through all long bridges. By item (5b) of Theorem 1, the defect $k$ group of $\Gamma$ as a permutation group is the direct product of the defect $k$ groups of $\Gamma_{1}^{\prime}, \ldots \Gamma_{l}^{\prime}$, and the defect $k$ groups of the trivial maximal $k$-subgraphs.

\section{Complexity of the Flow Semigroup of (Di)GRAPhS}

In this section we apply our results and the complexity lower bounds of [10] to verify [9, Conjecture 6.51i (1)] for 2-vertex connected graphs. That is, we prove that the Krohn-Rhodes (or group-) complexity of the flow semigroup of a 2-vertex connected graph with $n$ vertices is $n-2$ (item $\left(4^{\mathrm{c}}\right)$ of Theorem 11). Then we derive item $\left(4^{\mathrm{cc}}\right)$ of Theorem 1 as a further consequences of our results.

For standard definitions on wreath product of semigroups, we refer the reader to e.g. [9, Definition 2.2]. A finite semigroup $S$ is called combinatorial if and only if every maximal subgroup of $S$ has one element. Recall that the Krohn-Rhodes (or group-) complexity of a finite semigroup $S$ (denoted by $\#_{G}(S)$ ) is the smallest non-negative integer $n$ such that $S$ is a homomorphic image of a subsemigroup of the iterated wreath product

$$
C_{n} \prec G_{n} \prec \cdots \prec C_{1} \prec G_{1} \prec C_{0},
$$

where $G_{1}, \ldots, G_{n}$ are finite groups, $C_{0}, \ldots, C_{n}$ are finite combinatorial semigroups, and 2 denotes the wreath product (for the precise definition, see e.g. [9, Definition 3.13]). The definition immediately implies that if a finite semigroup $S$ is the homomorphic image of a subsemigroup of $T$, then $\#_{G}(S) \leq \#_{G}(T)$. More can be found on the complexity of semigroups in e.g. [9, Chapter 3]. We need the following results on the complexity of semigroups.

Lemma 25 ([9, Prop. 6.49(b)]). The flow semigroup $K_{n}$ of the complete graph on $n \geq 2$ vertices has $\#_{G}\left(K_{n}\right)=n-2$.

Lemma 26 ([10, Sec. 3.7]). The complexity of the full transformation semigroup $F_{n}$ on $n$ points is $\#_{G}\left(F_{n}\right)=n-1$.

The well-known $\mathcal{L}$-order is a pre-order, i.e. a transitive and reflexive binary relation, on the elements of a semigroup $S$ given by $s_{1} \succeq_{\mathcal{L}} s_{2}$ if $s_{1}=s_{2}$ or $s s_{1}=s_{2}$ for some $s \in S$. The $\mathcal{L}$-classes of $S$ are the equivalence classes of the $\mathcal{L}$-order. The $\mathcal{L}$-classes are thus partially ordered by $L_{1} \succeq_{\mathcal{L}} L_{2}$ if and only if $S L_{1} \cup L_{1} \supseteq S L_{2} \cup L_{2}$. One says that a finite semigroup $S$ is a $T_{1}$-semigroup if it is generated by some $\succeq_{\mathcal{L}^{-}}$-chain of its $\mathcal{L}$-classes, i.e. if there exist $\mathcal{L}$-classes $L_{1} \succeq_{\mathcal{L}} \cdots \succeq_{\mathcal{L}} L_{m}$ of $S$ such that $S=\left\langle L_{1} \cup \ldots \cup L_{m}\right\rangle$. Equivalently, $S$ is a $T_{1}$-semigroup if there exist $U_{i} \subseteq L_{i}(1 \leq i \leq m)$ for such a chain of $\mathcal{L}$-classes of $S$ such that $S=\left\langle U_{1} \cup \ldots \cup U_{m}\right\rangle$. 
Lemma 27 ([10, Lemma 3.5(b)]). Let $S$ be a noncombinatorial $T_{1}$ semigroup. Then

$$
\#_{G}(S) \geq 1+\#_{G}(E G(S))
$$

where $E G(S)$ is the subsemigroup of $S$ generated by all its idempotents.

Now we prove [9, Conjecture 6.51i (1)] for 2-vertex connected graphs.

Proof of item $\left(4^{c}\right)$ of Theorem 1 . Let $\Gamma$ be a 2-vertex connected simple graph with $n \geq 2$ vertices. Let $K_{n}$ denote the flow semigroup of the complete graph on vertices $V$, where $|V|=n$. Then $\#_{G}\left(S_{\Gamma}\right) \leq$ $\#_{G}\left(K_{n}\right)=n-2$ by Lemma 25. We proceed by induction on $n$. If $n \leq 3$, then $\Gamma$ is a complete graph, and $\#_{G}\left(S_{\Gamma}\right)=n-2$ by Lemma 25 . From now on we assume $n>3$ and $\Gamma=(V, E)$.

Case 1. Assume first that $\Gamma$ is not a cycle. Let $(u, v)$ and $(x, y)$ be two disjoint edges in $\Gamma$. Let $G_{1}$ be the defect 1 group with defect set $V \backslash\{u\}$ and idempotent $e_{u v}$ as its identity element. Then $e_{u v} \succeq_{\mathcal{L}}$ $e_{x y} e_{u v}=e_{u v} e_{x y}$. Let $T$ be $\left\langle G_{1} \cup\left\{e_{u v} e_{x y}\right\}\right\rangle$. Since $G_{1} \succeq_{\mathcal{L}}\left\{e_{u v} e_{x y}\right\}$ is an $\mathcal{L}$-chain in $T, T$ is a $T_{1}$-semigroup. Furthermore, $T$ is noncombinatorial since $G_{1}$ is nontrivial. Thus, by Lemma 27

$$
\#_{G}(T) \geq 1+\#_{G}(E G(T)) .
$$

Let $\Gamma^{\prime}$ be the complete graph on $V \backslash\{u\}$. Let $a, b \in V \backslash\{u\}$ be arbitrary distinct vertices. By item (4) of Theorem 1, $G_{1}$ is 2-transitive. Let $\pi \in G_{1}$ be such that $\pi(x)=a$ and $\pi(y)=b$. There is a positive integer $\omega>1$, with $\pi^{\omega}=e_{u v}$. In particular, $e_{u v}$ commutes with $\pi$. Observe that

$$
\begin{aligned}
& \pi^{\omega-1} e_{u v} e_{x y} \pi=e_{u v}\left(\pi^{\omega-1} e_{x y} \pi\right)=e_{u v} e_{a b}, \text { and thus } \\
& \left(\pi^{\omega-1} e_{x y} e_{u v} \pi\right)\left\lceil_{V \backslash\{u\}}=e_{a b} .\right.
\end{aligned}
$$

That is, we obtain the generators $e_{a b}$ of $S_{\Gamma^{\prime}}$ by restricting the idempotents $e_{u v} e_{a b} \in T$ to $V \backslash\{u\}$. Therefore, $S_{\Gamma^{\prime}}$ is a homomorphic image of a subsemigroup of $E G(T)$, yielding

$$
\#_{G}(E G(T)) \geq \#_{G}\left(S_{\Gamma^{\prime}}\right) .
$$

By induction, $\#_{G}\left(S_{\Gamma^{\prime}}\right)=n-3$. Applying (11), we obtain $\#_{G}(T) \geq$ $n-2$. Since $T$ is a subsemigroup of $S_{\Gamma}$, we obtain $\#_{G}\left(S_{\Gamma}\right) \geq \#_{G}(T) \geq$ $n-2$.

Case 2. Assume now that $\Gamma$ is the $n$-node cycle $\left(u, v_{1}, \ldots, v_{n-1}\right)$. Then $\left(u, v_{1}\right)$ and $\left(v_{2}, v_{3}\right)$ are disjoint edges. Let $G_{1} \simeq Z_{n-1}$ be the defect 1 group with defect set $V \backslash\{u\}$ and idempotent $e_{u v_{1}}$ as its identity element. Let $\pi$ be a generator of $G_{1}$ with cycle structure $\left(v_{1}, \ldots, v_{n-1}\right)$. Then $e_{u v_{1}} \succeq_{\mathcal{L}} e_{v_{2} v_{3}} e_{u v_{1}}=e_{u v_{1}} e_{v_{2} v_{3}}$. Let $T$ be $\left\langle G_{1} \cup\left\{e_{u v_{1}} e_{v_{2} v_{3}}\right\}\right\rangle$. Since $G_{1} \succeq_{\mathcal{L}}\left\{e_{u v_{1}} e_{v_{2} v_{3}}\right\}$ is an $\mathcal{L}$-chain in $T, T$ is a $T_{1}$-semigroup. Furthermore, $T$ is noncombinatorial since $G_{1}$ is nontrivial. Thus, by Lemma 27

$$
\#_{G}(T) \geq 1+\#_{G}(E G(T)) .
$$


Let $\Gamma^{\prime}$ be an $(n-1)$-node cycle with nodes $V \backslash\{u\}=\left\{v_{1}, \ldots, v_{n-1}\right\}$. Note that $e_{u v_{1}}=\pi^{n-1}$, and therefore $e_{u v_{1}}$ commutes with $\pi$. Let $v_{i-1}, v_{i}, v_{i+1} \in V \backslash\{u\}$ be three neighboring nodes in $\Gamma^{\prime}$, where the indices are in $\{1, \ldots, n-1\}$ taken modulo $n-1$. Observe that

$$
\begin{aligned}
\pi^{n-2} e_{u v_{1}} e_{v_{i-1} v_{i}} \pi=e_{u v_{1}}\left(\pi^{n-2} e_{v_{i-1} v_{i}} \pi\right) & =e_{u v_{1}} e_{v_{i} v_{i+1}}, \text { and thus } \\
\left(\pi^{n-2} e_{u v_{1}} e_{v_{i-1} v_{i}} \pi\right)\left\lceil_{V \backslash\{u\}}\right. & =e_{v_{i} v_{i+1}} .
\end{aligned}
$$

That is, we obtain the generators $e_{v_{i} v_{i+1}}$ of $S_{\Gamma^{\prime}}$ by restricting the idempotents $e_{u v_{1}} e_{v_{i} v_{i+1}} \in T$ to $V \backslash\{u\}$. Therefore, $S_{\Gamma^{\prime}}$ is a homomorphic image of a subsemigroup of $E G(T)$, yielding

$$
\#_{G}(E G(T)) \geq \#_{G}\left(S_{\Gamma^{\prime}}\right) .
$$

By induction, $\#_{G}\left(S_{\Gamma^{\prime}}\right)=n-3$. Applying (2), we obtain $\#_{G}(T) \geq$ $n-2$. Since $T$ is a subsemigroup of $S_{\Gamma}$, we have $\#_{G}\left(S_{\Gamma}\right) \geq \#_{G}(T) \geq$ $n-2$.

Note that by Lemma 4 a strongly connected digraph has the same flow semigroup as the corresponding graph. Thus, item $\left(4^{\mathrm{c}}\right)$ of Theorem 1 proves Rhodes's conjecture [9, Conjecture 6.51i (1)] for 2-vertex connected strongly connected digraphs, as well. The following lemma bounds the complexity in the remaining cases.

Lemma 28. Let $k$ be the smallest positive integer such that for a graph $\Gamma$ the flow semigroup $S_{\Gamma}$ has defect $k$ group $S_{n-k}$. Then $\#_{G}\left(S_{\Gamma}\right) \geq$ $n-1-k$.

Proof. Assume first $k=n-1$. Then the lemma holds trivially. From now on, assume $k \leq n-2$. Let $u v$ be an edge in $\Gamma$. Let $V_{k}$ be an arbitrary $k$-element subset of the vertex set $V$ disjoint from $\{u, v\}$. Let $G_{k}$ be the defect $k$ group with defect set $V_{k}$. Let $S$ be the subsemigroup of $S_{\Gamma}$ generated by $G_{k}$ and $e_{u v}$. As $G_{k} \simeq S_{n-k}$, we have that $S$ is the semigroup of all transformations on $V \backslash V_{k}$. Hence, $\#_{G}(S)=$ $\#_{G}\left(F_{n-k}\right)=n-k-1$ by Lemma 26. Whence, $\#_{G}\left(S_{\Gamma}\right) \geq \#_{G}(S)=$ $n-k-1$.

By Theorem 19, it immediately follows that the complexity of the flow semigroup of a 2-edge connected graph $\Gamma$ is at least $n-3$. Furthermore, $\#_{G}\left(S_{\Gamma}\right) \leq \#_{G}\left(K_{n}\right)=n-2$ by Lemma 25. This finishes the proof of item $\left(4^{\mathrm{cc}}\right)$ of Theorem 1 .

\section{REFERENCES}

[1] P. J. Cameron. Permutation Groups, volume 45 of London Mathematical Society Student Texts. Cambridge University Press, Cambridge, 1999.

[2] A. H. Clifford and G. B. Preston. The Algebraic Theory of Semigroups. Vol. I. Number 7 in Mathematical Surveys. American Mathematical Society, Providence, R.I., 1961. 
[3] A. H. Clifford and G. B. Preston. The Algebraic Theory of Semigroups. Vol. II. Number 7 in Mathematical Surveys. American Mathematical Society, Providence, R.I., 1967.

[4] R. Diestel. Graph Theory, volume 173 of Graduate Texts in Mathematics. Springer, Heidelberg, fourth edition, 2010.

[5] J. D. Dixon and B. Mortimer. Permutation Groups, volume 163 of Graduate Texts in Mathematics. Springer-Verlag, New York, 1996.

[6] J. East, M. Gadouleau, and J. D. Mitchell. On transformation semigroups based on digraphs. 2017. submitted, https://arxiv.org/abs/1704.00937

[7] R. L. Graham, M. Grötschel, and L. Lovász, editors. Handbook of Combinatorics. Vol. 1, 2. Elsevier Science B.V., Amsterdam; MIT Press, Cambridge, MA, 1995.

[8] J. Hopcroft and R. Tarjan. Algorithm 447: Efficient algorithms for graph manipulation. Commun. ACM, 16(6):372-378, June 1973.

[9] J. Rhodes. Applications of Automata Theory and Algebra: Via the Mathematical Theory of Complexity to Biology, Physics, Psychology, Philosophy, and Games. World Scientific Publishing Co. Pte. Ltd., Singapore, 2010. Edited by Chrystopher L. Nehaniv, with a foreword by Morris W. Hirsch. [Original Version: University of California at Berkeley Mathematics Library, 1971].

[10] J. Rhodes and B. R. Tilson. Lower bounds for complexity of finite semigroups. J. Pure Appl. Algebra, 1(1):79-95, 1971.

[11] H. E. Robbins. Questions, Discussions, and Notes: A Theorem on Graphs, with an Application to a Problem of Traffic Control. Amer. Math. Monthly, 46(5):281-283, 1939.

[12] D. J. S. Robinson. A Course in the Theory of Groups, volume 80 of Graduate Texts in Mathematics. Springer-Verlag, New York, second edition, 1996.

[13] R. E. Tarjan. A note on finding the bridges of a graph. Information Processing Lett., 2:160-161, 1973/74.

[14] H. Whitney. Non-separable and planar graphs. Trans. Amer. Math. Soc., 34(2):339-362, 1932.

[15] R. M. Wilson. Graph puzzles, homotopy, and the alternating group. J. Combinatorial Theory Ser. B, 16:86-96, 1974.

Institute of Mathematics, University of Debrecen, Pf. 400, DebreCEN, 4002, HUNGARY

E-mail address: ghorvath@science.unideb.hu

Royal Society / Wolfson Foundation Biocomputation Research Laboratory, Centre for Computer Science and Informatics Research, University of Hertfordshire, College Lane, Hatfield, Hertfordshire Al10 9AB, United Kingdom

E-mail address: C.L.Nehaniv@herts.ac.uk

Alfréd Rényi Institute of Mathematics, 13-15 ReÁltanoda utca, BuDAPEST, 1053, HUNGARY

E-mail address: pcharles@renyi.mta.hu 